\title{
Online drift estimation for jump-diffusion
}

\section{processes}

\author{
THEERAWAT BHUDISAKS ANG* and ÁLVARO CARTEA ${ }^{\dagger}$ \\ Mathematical Institute, University of Oxford, Woodstock Rd, Oxford, OX2 6GG, UK. \\ E-mail: ${ }^{*}$ theerawat.bhudisaksang@maths.ox.ac.uk; ${ }^{\dagger}$ alvaro.cartea@maths.ox.ac.uk
}

\begin{abstract}
We show the convergence of an online stochastic gradient descent estimator to obtain the drift parameter of a continuous-time jump-diffusion process. The stochastic gradient descent follows a stochastic path in the gradient direction of a function to find a minimum, which in our case determines the estimate of the unknown drift parameter. We decompose the deviation of the stochastic descent direction from the deterministic descent direction into four terms: the weak solution of the non-local Poisson equation, a Riemann integral, a stochastic integral, and a covariation term. This decomposition is employed to prove the convergence of the online estimator and we use simulations to illustrate the performance of the online estimator.
\end{abstract}

Keywords: SGDCT; online estimation; jump-diffusion; extended Itô lemma; non-local Poisson equation; Lévy process

\section{Introduction}

Typically, one judges the usefulness of models by how good they are in describing a system and by the accuracy of their predictions. In both cases, the degree of success of a model depends on a number of ingredients, which include: model assumptions, mathematical tractability, and the methodology to estimate the parameters of the model. In this paper, we assume that the system is a jump-diffusion process and we show how to estimate its drift component with a learning algorithm that updates the parameter estimates in continuous-time. Specifically, one observes the process $X=\left(X_{t}\right)_{t \geq 0}$ that takes values in $\mathbb{R}^{n}$, and which satisfies the stochastic differential equation (SDE)

$$
d X_{t}=b\left(X_{t}, \theta^{*}\right) d t+\sigma\left(X_{t}\right) d B_{t}+\xi\left(X_{t-}, d L_{t}\right),
$$

where the jump component $\xi\left(X_{t-}, d L_{t}\right)$ is given by

$$
\xi\left(X_{t-}, d L_{t}\right)=\int_{0}^{t} \int \xi\left(X_{t-}, z\right) \tilde{\mu}(d t, d z) .
$$

Here, $\theta^{*} \in \mathbb{R}^{d}$ is an unknown parameter that one needs to estimate from observations of the process $X$. The process $B=\left(B_{t}\right)_{t \geq 0}$ is a standard $m$-dimensional Brownian motion and the functions $b$, $\sigma, \xi$ are such that $b: \mathbb{R}^{n} \times \mathbb{R}^{\bar{d}} \rightarrow \mathbb{R}^{n}, \sigma: \mathbb{R}^{n} \rightarrow \mathbb{R}^{n} \times \mathbb{R}^{m}, \xi: \mathbb{R}^{n} \times \mathbb{R}^{r} \rightarrow \mathbb{R}^{n}$. In the jump component of $X_{t}, \tilde{\mu}$ is a compensated random measure of the Poisson measure $\mu$ associated with the pure-jump Lévy process $L=\left(L_{t}\right)_{t \geq 0}$ (independent of $B$ ) with Lévy-Khintchine triplet $(0,0, v)$, such that $\int_{\mathbb{R}^{r}}|z| v(d z)<\infty$ and $v\left(\mathbb{R}^{r}\right)<\infty$.

Here is a brief summary of our approach to estimate the parameter $\theta^{*}$. We denote by $\langle\cdot, \cdot\rangle$ the dot product operator and define the function $\bar{g}(\theta):=\int g(x, \theta) \pi^{\theta^{*}}(d x)$, where $\pi^{\theta^{*}}$ is a unique invariant measure of the process $X$ under the probability measure $\mathbb{P}_{\theta^{*}}$, and where

$$
g(x, \theta):=\frac{1}{2}\left\langle b(x, \theta)-b\left(x, \theta^{*}\right),\left(\sigma(x) \sigma(x)^{\top}\right)^{-1}\left(b(x, \theta)-b\left(x, \theta^{*}\right)\right)\right\rangle
$$


is a function that specifies how close the function $b(x, \theta)$ is to the drift component $b\left(x, \theta^{*}\right)$ of the process $X$.

Next, we employ the deterministic gradient descent method to construct a process $\theta_{t}$ that converges to a stationary point of the function $\bar{g}$. Recall that the deterministic descent direction $\nabla_{\theta} \bar{g}(\theta)$ moves the value of the parameter $\theta$ toward a stationary point (local or global) of the function $\bar{g}$. This stationary point is the estimate of the unknown parameter $\theta^{*}$. In continuous-time, the updates of the gradient descent to a stationary point of the function $\bar{g}$ follow

$$
\begin{aligned}
d \theta_{t}= & -\beta_{t} \nabla_{\theta} \bar{g}\left(\theta_{t}\right) d t \\
= & -\beta_{t} \nabla_{\theta} g\left(X_{t}, \theta_{t}\right) d t+\beta_{t}\left(\nabla_{\theta} g\left(X_{t}, \theta_{t}\right)-\nabla_{\theta} \bar{g}\left(\theta_{t}\right)\right) d t \\
= & \beta_{t}\left(\nabla_{\theta} b\left(X_{t}, \theta_{t}\right)\right)\left(\sigma\left(X_{t}\right) \sigma\left(X_{t}\right)^{\top}\right)^{-1}\left(b\left(X_{t}, \theta^{*}\right)-b\left(X_{t}, \theta_{t}\right)\right) d t \\
& +\beta_{t}\left(\nabla_{\theta} g\left(X_{t}, \theta_{t}\right)-\nabla_{\theta} \bar{g}\left(\theta_{t}\right)\right) d t,
\end{aligned}
$$

where $\beta_{t}>0$ is the learning rate.

One cannot implement the unbiased estimator in (1.4) because the drift term $b\left(x, \theta^{*}\right)$ depends on the unknown value of $\theta^{*}$. Thus, we employ (1.1) to write

$$
b\left(X_{t}, \theta^{*}\right) d t=d X_{t}-\int \xi\left(X_{t^{-}}, z\right) \tilde{\mu}(d z, d t)-\sigma\left(X_{t}\right) d B_{t},
$$

which we substitute into (1.4), so the estimator

$$
\begin{aligned}
d \theta_{t}= & \beta_{t}\left[\nabla_{\theta} b\left(X_{t}, \theta_{t}\right)\left(\sigma\left(X_{t}\right) \sigma\left(X_{t}\right)^{\top}\right)^{-1} d X_{t}-\nabla_{\theta} b\left(X_{t}, \theta_{t}\right)\left(\sigma\left(X_{t}\right) \sigma\left(X_{t}\right)^{\top}\right)^{-1} b\left(X_{t}, \theta_{t}\right) d t\right] \\
& +\beta_{t}\left(\nabla_{\theta} g\left(X_{t}, \theta_{t}\right)-\nabla_{\theta} \bar{g}\left(\theta_{t}\right)\right) d t-\beta_{t} \nabla_{\theta} b\left(X_{t}, \theta_{t}\right)\left(\sigma\left(X_{t}\right) \sigma\left(X_{t}\right)^{\top}\right)^{-1} \sigma\left(X_{t}\right) d B_{t} \\
& -\beta_{t} \nabla_{\theta} b\left(X_{t}, \theta_{t}\right)\left(\sigma\left(X_{t}\right) \sigma\left(X_{t}\right)^{\top}\right)^{-1} \int \xi\left(X_{t-}, z\right) \tilde{\mu}(d t, d z)
\end{aligned}
$$

does not depend on the unknown parameter $\theta^{*}$.

In this paper, we show that the integrals

$$
\int_{t}^{\infty} \beta_{s}\left(\nabla_{\theta} g\left(X_{s}, \theta_{s}\right)-\nabla_{\theta} \bar{g}\left(\theta_{s}\right)\right) d s
$$

and

$$
\begin{aligned}
\int_{t}^{\infty} & \beta_{s} \nabla_{\theta} b\left(X_{s}, \theta_{s}\right)\left(\sigma\left(X_{s}\right) \sigma\left(X_{s}\right)^{\top}\right)^{-1} \sigma\left(X_{s}\right) d B_{s} \\
+ & \int_{t}^{\infty} \beta_{s} \nabla_{\theta} b\left(X_{s}, \theta_{s}\right)\left(\sigma\left(X_{s}\right) \sigma\left(X_{s}\right)^{\top}\right)^{-1} \int \xi\left(X_{s-}, z\right) \tilde{\mu}(d s, d z)
\end{aligned}
$$

converge to zero as $t \rightarrow \infty$. Therefore, the expression in (1.6) is close to the deterministic descent direction $\nabla_{\theta} \bar{g}$ when $t$ is large because the contribution of the last three terms in (1.6) becomes negligible. Hence, in this paper we propose the stochastic gradient descent in continuous-time (SGDCT) algorithm

$$
d \theta_{t}=\beta_{t}\left[\nabla_{\theta} b\left(X_{t}, \theta_{t}\right)\left(\sigma\left(X_{t}\right) \sigma\left(X_{t}\right)^{\top}\right)^{-1} d X_{t}-\nabla_{\theta} b\left(X_{t}, \theta_{t}\right)\left(\sigma\left(X_{t}\right) \sigma\left(X_{t}\right)^{\top}\right)^{-1} b\left(X_{t}, \theta_{t}\right) d t\right]
$$

to estimate the unknown drift parameter $\theta^{*}$. 
One can see how the SGDCT estimator compares to the classical maximum likelihood estimator (MLE) when the process $X$ is a diffusion. Above, if one excludes the jump component in (1.1), the process $X$ becomes a diffusion, in which case the estimate of the parameter $\theta$ is the value that maximises the likelihood function

$$
\mathcal{L}_{t}(\theta)=\int_{0}^{t} b\left(X_{s}, \theta\right)\left(\sigma\left(X_{t}\right) \sigma\left(X_{t}\right)^{\top}\right)^{-1} d X_{s}-\frac{1}{2} \int_{0}^{t} b\left(X_{s}, \theta\right)\left(\sigma\left(X_{t}\right) \sigma\left(X_{t}\right)^{\top}\right)^{-1} b\left(X_{s}, \theta\right) d s .
$$

Observe that the first order condition one obtains from maximising the likelihood (i.e., computing the gradient of $\mathcal{L}_{t}(\theta)$ with respect to $\theta$ and equating to zero) has the same form as the expression that appears inside the brackets in the first line of the estimator in (1.9).

There are two reasons that make a recursive estimator like that in (1.9) useful. First, online updates of the estimator are more computationally efficient than those based on offline updates. As new observations arrive, the online estimator is updated, while the offline algorithm solves an new optimisation problem to estimate the unknown parameter; for example, find the maximiser of the MLE in (1.10). Therefore, the offline algorithm is in most cases redundant and inefficient, and in high dimensions it is computationally expensive and in some cases infeasible.

Second, the SGDCT estimator is well-suited for the recent advances in control theory that focus on 'adaptive robust control' to obtain strategies robust to model uncertainty, see Bielecki et al. [3]. The adaptive control framework requires a recursive online estimator (i.e., an estimator that is continuously updated with the arrival of new information) and also requires that the coupled process $\left(X_{t}, \theta_{t}\right)_{t \geq 0}$ is Markov (to obtain the optimal strategy in feedback form). The SGDCT algorithm in (1.9) satisfies both requirements.

Finally, there is literature on offline estimators for diffusion and jump-diffusion processes that focuses on different aspects of the estimation algorithm and observation frequency. When an ergodic diffusion process is observed continuously, Kutoyants [10] derives the statistical properties of the estimator of the drift parameter, such as consistency, asymptotic normality, and efficiency. These results are extended in Sorensen [19] for an ergodic jump-diffusion process. The literature on parameter estimation with discrete observations is vast, see, for example, Yoshida [24] for diffusions and Kessler [8] for jump-diffusions.

On the other hand, the literature on parameter estimation for continuously observed processes is scant. In Levanony [11], the authors assume that the process $X$ follows a diffusion and develop an online MLE to obtain the drift parameter of the process $X$. In their approach, the online MLE requires one to compute the gradient of $\mathcal{L}_{t}(\theta)$ in (1.10) every time a new observation arrives, which is computationally expensive because the function $\nabla_{\theta} \mathcal{L}_{t}$ depends on the whole trajectory of the process $X$. Moreover, there are two papers that discuss the convergence of an online estimation of parameters in a diffusion-based model. Sirignano and Spiliopoulos [17] introduce an online method to estimate parameters of a continuously observed diffusion, that is, the process follows (1.1) without the jump component $\xi\left(X_{t-}, d L_{t}\right)$; thus, our paper extends their work by including jumps in the stochastic process. The work of Surace and Pfister [20] studies the problem in [17] when the process $X$ is a diffusion (i.e., no jumps) and it is partially observed.

The remainder of the paper is organised as follows. In Section 2, we state conditions on the functions $b, \sigma, \xi$, so that the process $X$ in (1.1) is exponentially ergodic. Then, we show that the term $\int_{t}^{\infty} \beta_{s}\left(\nabla_{\theta} g\left(X_{s}, \theta_{s}\right)-\nabla_{\theta} \bar{g}\left(\theta_{s}\right)\right) d s$ converges to zero as $t \rightarrow \infty$, and we prove the convergence of $\theta_{t}$ to a stationary point of the function $\bar{g}$. Section 3 concludes and discusses extensions and applications of our work. Finally, in the online supplement [2] we provide additional proofs and material, including a study of the performance of SGDCT. 


\section{Online recursive estimator}

In this section, we state the assumptions that we use throughout this paper and show our main results. We recall that the function

$$
g(x, \theta)=\frac{1}{2}\left\langle b(x, \theta)-b\left(x, \theta^{*}\right),\left(\sigma(x) \sigma(x)^{\top}\right)^{-1}\left(b(x, \theta)-b\left(x, \theta^{*}\right)\right)\right\rangle,
$$

specifies how close the function $b(x, \theta)$ is to the drift $b\left(x, \theta^{*}\right)$ of the process $X$. Note that the function $g(x, \theta) \geq 0$, and $g(x, \theta)=0$ if $\theta=\theta^{*} .1$

A pivotal result in this paper is to show that

$$
\left|\Gamma_{k, \gamma}\right| \rightarrow 0 \quad \text { as } k \rightarrow \infty, \text { almost surely, }
$$

where

$$
\Gamma_{k, \gamma}:=\int_{\underline{\tau}_{k}}^{\bar{\tau}_{k, \gamma}} \beta_{s}\left(\nabla_{\theta} g\left(X_{s}, \theta_{s}\right)-\nabla_{\theta} \bar{g}\left(\theta_{s}\right)\right) d s
$$

is a deviation term. Here, the processes $\left\{\bar{\tau}_{k, \gamma}\right\}_{k \geq 1},\left\{\underline{\tau}_{k}\right\}_{k \geq 1}$ are sequences of increasing stopping times and $|\theta|$ denotes the Euclidean norm of $\theta \in \mathbb{R}^{d}$, and recall that $\bar{g}(\theta)=\int g(x, \theta) \pi^{\theta^{*}}(d x)$. We refer to $\Gamma_{k, \gamma}$ in (2.3) as the deviation term because it represents the deviation of the stochastic gradient descent direction $\nabla_{\theta} g\left(X_{s}, \theta_{s}\right)$ from the deterministic descent direction $\nabla_{\theta} \bar{g}\left(\theta_{s}\right)$. Therefore, we need a careful analysis of the deviation term $\Gamma_{k, \gamma}$ to show that $\theta_{t}$ converges to the unknown parameter $\theta^{*}$.

We show that the deviation term $\Gamma_{k, \gamma}$ can be written as a sum of four components: a weak solution of a non-local Poisson equation, a stochastic integral, a Riemann integral, and the covariation of two processes. Then, we prove that each component converges to zero as $k \rightarrow \infty$, so the estimator process $\theta_{t}$ converges to a stationary point of the function $\bar{g}$. That is, we show that $\lim _{t \rightarrow \infty}\left|\nabla_{\theta} \bar{g}\left(\theta_{t}\right)\right|=0$ almost surely, and if the function $\bar{g}(\theta)$ is convex, the minimum we find is global, hence $\theta_{t} \rightarrow \theta^{*}$ as $t \rightarrow \infty$.

\subsection{Main result and conditions}

To streamline the discussion and results, we provide some preliminary assumptions. Throughout this paper, the stochastic process $X$ satisfies the SDE in (1.1).

Condition 2.1. The functions $\sigma(x)$ and $\xi(x, z)$ are globally Lipschitz in all variables and the function $b(x, \theta)$ is locally Lipschitz for all $\theta$.

Condition 2.2. There exists a time $t>0$, such that $X_{t}$ admits a density $p_{t}^{\theta}(x, y)$ with respect to the Lebesgue measure on $\mathbb{R}$, bounded in $y \in \mathbb{R}$, and bounded in $x \in K$ for every compact set $K \subset \mathbb{R}$. Moreover, for every $x \in \mathbb{R}$, and every open ball $U \in \mathbb{R}$, there exists a point $z=z(x, U) \in \operatorname{supp}(v)$, where $\operatorname{supp}(v)$ is the support of the measure $v$ (see definition 4.5 in online supplement [2]), such that $\xi(x, z) \in U$.

\footnotetext{
${ }^{1}$ One could consider alternative functions to $g$ to specify how close is $b(x, \theta)$ to $b\left(x, \theta^{*}\right)$. For example (in the one dimensional case), let $h(x, \theta):=\frac{1}{2}\left|\left(c(x, \theta)-c\left(x, \theta^{*}\right)\right) / \sigma(x)\right|^{2}$, where $c$ is an arbitrary function and $\bar{h}(\theta)=\int h(x, \theta) \pi^{\theta^{*}}(d x)$. Then, as in (1.4), the estimator of the unknown parameter would be given by $d \theta_{t}=-\beta_{t} \nabla_{\theta} \bar{h}\left(\theta_{t}\right) d t$. As discussed above, the drift term $c\left(x, \theta^{*}\right)$ depends on the unknown value of $\theta^{*}$, so one needs to employ the dynamics of $d X_{t}$ to write $b\left(X_{t}, \theta^{*}\right)$ and substitute it in the estimator. Thus, the function $c$ should be such that one can factor out the term $b\left(X_{t}, \theta^{*}\right)$; for example, $c(x, \theta)=d(x) b(x, \theta)$ for a function $d(x)$.
} 


\section{Condition 2.3. Exponential ergodicity of the process $X$.}

(i) For all $p>0, \int_{|z|>1}|z|^{p} v(d z)<\infty$ and $v\left(\mathbb{R}^{r}\right)<\infty$.

(ii) For all $\theta$ there exists a constant $C>0$, such that $x b(x, \theta) \leq-C|x|^{2}$ as $|x| \rightarrow \infty$.

(iii) $|\xi(x, z)| /|x| \rightarrow 0$ as $|x| \rightarrow \infty$ for all $z$.

(iv) There exist positive constants $C_{1}$ and $C_{2}$, s.t. $C_{1} \leq \sigma_{i j}^{2}(x) \leq C_{2}$ for all $1 \leq i \leq n$ and $1 \leq j \leq m$ where $\sigma_{i j}$ denote the entry of row $i$ and column $j$ of the matrix $\sigma$.

Under Conditions 2.1 and 2.3, the SDE in (1.1) admits a unique non-explosive cádlág adapted solution that possesses the strong Markov property. Masuda [12] employs Condition 2.2 to show that the process $X$ is irreducible; a property required to show that $X$ is ergodic. Also, $X$ has a unique invariant measure and it is exponentially ergodic due to Condition 2.3. Finally, the process $X$ is of finite jump activity because $v$ is a finite measure, so there is a finite number of jumps on every compact interval almost surely.

Condition 2.4. The learning rate $\beta_{t}$ is such that the following hold: $\int_{0}^{\infty} \beta_{t} d t=\infty, \int_{0}^{\infty} \beta_{t}^{2} d t<\infty$, $\int_{0}^{\infty}\left|\beta_{t}^{\prime}\right| d t<\infty$, where $\beta_{t}^{\prime}$ is the derivative of $\beta_{t}$ with respect to $t$, and there is a $p>0$, such that $\lim _{t \rightarrow \infty} \beta_{t}^{2} t^{2 p+1 / 2}=0$.

Condition 2.5. The function $b(x, \theta)$ is twice-differentiable with respect to both $x$ and $\theta$. Also, there exist positive constants $K$ and $q$, such that

$$
\left|\frac{\partial \nabla_{\theta} g}{\partial x}(x, \theta)\right|+\left|\nabla_{\theta} g(x, \theta)\right| \leq K(1+|\theta|)\left(1+|x|^{q}\right),
$$

and

$$
\sum_{i=1}^{2}\left|\frac{\partial^{i} \nabla_{\theta} g}{\partial \theta^{i}}(x, \theta)\right|+\left|\nabla_{\theta} b(x, \theta)\right| \leq K\left(1+|x|^{q}\right) .
$$

Moreover, we assume that $\nabla_{\theta} \bar{g}(\theta)$ is globally Lipschitz.

Condition 2.6. There exists a positive function $\kappa(x)$, such that

$$
\left\langle-\nabla_{\theta} g(x, \theta), \theta /|\theta|\right\rangle \leq-\kappa(x)|\theta|
$$

Thus, there exists a function $\lambda(x)$ with at most polynomial growth in $|x|$, such that for all $\theta_{1}, \theta_{2} \in \mathbb{R}^{d}$ and $x \in \mathbb{R}^{n}$, we have

$$
\left|\tau\left(x, \theta_{1}\right)-\tau\left(x, \theta_{2}\right)\right| \leq|\lambda(x)|\left|\theta_{1}-\theta_{2}\right|
$$

where

$$
\tau(x, \theta):=\left\langle\nabla_{\theta} b(x, \theta) \nabla_{\theta} b(x, \theta)^{\top} \theta /|\theta|, \theta /|\theta|\right\rangle^{1 / 2} .
$$

Lastly, for all $x_{2} \geq x_{1}$, we have $\xi\left(x_{1}, z\right)-\xi\left(x_{2}, z\right) \leq x_{2}-x_{1}$, where the greater or equal symbol $\geq$ indicates the inequality holds for all coordinates.

Condition 2.4 ensures that the size of the learning rate $\beta$ is such that the SGDCT estimator for the jump-diffusion process in (1.9) converges to a stationary point of the function $\bar{g}(\theta)$. Condition 2.5 is needed for the proof of the convergence of the deviation term $\Gamma_{k, \gamma}$, that is, $\Gamma_{k, \gamma} \rightarrow 0$ as $k \rightarrow \infty$, see (2.2). Condition 2.6 allows us to use a comparison theorem to show that the bound $\sup _{t>0} \mathbb{E}\left[\left|\theta_{t}\right|^{p}\right]<\infty$ for all $p>0$. 


\subsection{Weak solution of a non-local Poisson equation}

In this subsection, we define a notion of a weak solution of a Poisson equation to construct a weak solution of the process $X$. The following terminology is from Veretennikov and Kulik [22].

Definition 2.7. For a Markov process $X$, we say that a measurable function $f: \mathbb{R}^{n} \rightarrow \mathbb{R}^{d}$ belongs to the domain of the extended generator $\mathcal{A}$ of the process $X$ if there exists a measurable function $g: \mathbb{R}^{n} \rightarrow \mathbb{R}^{d}$, such that the process $f\left(X_{t}\right)-\int_{0}^{t} g\left(X_{s}\right) d s$ is a local $\mathbb{F}^{X}$-martingale. Then, we say that the function $f$ is the weak solution of the Poisson equation $\mathcal{A} f=g$.

We also need the following notation. The $f$-norm is denoted by

$$
\|\omega\|_{f}:=\sup _{|g| \leq f}\left|\int g(y) \omega(d y)\right|,
$$

where $\omega$ is a signed measure and $f \geq 1$ is a Borel measurable function. Also, we say that the function $g$ is centered if $\int g(x, \theta) \pi^{\theta^{*}}(d x)=0$.

Next, we state an auxiliary lemma to show the ergodicity of the process $X$ and to show the existence of moments of all orders for the processes $X$ and $\theta$.

Lemma 2.8 (Ergodicity and moment bounds of the processes $X$ and $\theta$ ). Suppose that Conditions 2.1 to 2.5 hold. Then, there exists a unique invariant measure $\pi^{\theta^{*}}$, such that $\int|x|^{p} \pi^{\theta^{*}}(d x)<\infty$ and there exist positive constants $C_{1}$ and $C_{2}$, such that

$$
\left\|\mathbb{P}_{\theta^{*}, t}(x, \cdot)-\pi^{\theta^{*}}(\cdot)\right\|_{1+|x|^{p}} \leq C_{1}\left(1+|x|^{p}\right) e^{-C_{2} t}
$$

for all $p>0, x \in \mathbb{R}^{n}$ and $t>0$. Thus, $\sup _{t>0} \mathbb{E}_{x, \theta^{*}}\left[\left|X_{t}\right|^{p}\right]<\infty$ for all $p>0$. In addition, if Condition 2.6 holds, we have that

$$
\sup _{t>0} \mathbb{E}_{x, \theta^{*}}\left[\left|\theta_{t}\right|^{p}\right]<\infty \text { for all } p>0
$$

For a proof, see Section 4 in the online supplement [2].

Now, we state our first theorem which is important to show that the deviation term $\Gamma_{k, \gamma} \rightarrow 0$ as $k \rightarrow \infty$, see (2.2).

Theorem 2.9 (Weak Poisson equation). Let the process $X$ follow (1.1). Suppose that Conditions 2.1 to 2.5 hold. Let $f$ be a centered function (i.e., $\int f(x, \theta) \pi^{\theta^{*}}(d x)=0$ for all $\theta$ ) and assume that for each $\theta$, there exist positive constants $C_{\theta}$ and $q$, such that $|f(x, \theta)| \leq C_{\theta}\left(1+|x|^{q}\right)$ for all $x$. Then, the function

$$
F(x, \theta):=\int_{0}^{\infty} \mathbb{E}_{x, \theta^{*}}\left[f\left(X_{t}, \theta\right)\right] d t
$$

is well-defined and satisfies, in a weak sense, the Poisson equation

$$
\mathcal{A}_{x, \theta^{*}} F(x, \theta)=-f(x, \theta),
$$

where $\mathcal{A}_{x, \theta^{*}}$ is the extended generator of the process $X$ under the probability measure $\mathbb{P}_{\theta^{*}}$ (see Definition 2.7). 
Proof. First, we show that the function $F(x, \theta)$ is well-defined. Recall that $\mathbb{E}_{x, \theta^{*}}\left[f\left(X_{t}, \theta\right)\right]=$ $\int f(y, \theta) p_{t}\left(x, d y ; \theta^{*}\right)$ and that $p_{t}\left(x, d y ; \theta^{*}\right)$ is the distribution of $X_{t}$ under the probability measure $\mathbb{P}_{\theta^{*}}$ and $X_{0}=x$. Note that

$$
\begin{aligned}
\left|\mathbb{E}_{x, \theta^{*}}\left[f\left(X_{t}, \theta\right)\right]\right| & =\left|\int f(y, \theta) p_{t}\left(x, d y ; \theta^{*}\right)-\int f(y, \theta) \pi^{\theta^{*}}(d y)\right| \\
& \leq C_{1}\left(1+|x|^{q}\right) e^{-C_{2} t} .
\end{aligned}
$$

Next, we show that the function $F$ is the solution to the Poisson equation in (2.12). It suffices to show that for all $\theta$, the process $F\left(X_{t}, \theta\right)+\int_{0}^{t} f\left(X_{s}, \theta\right) d s$ is an $\mathbb{F}$-martingale under the probability measure $\mathbb{P}_{\theta^{*}}$, as in the definition of the weak solution of the Poisson equation. Thus, we first show that the first moment of $F\left(X_{t}, \theta\right)+\int_{0}^{t} f\left(X_{s}, \theta\right) d s$ is finite for all $t$.

From (2.11) and (2.13), we have that $F\left(X_{t}, \theta\right) \leq\left(C_{1} / C_{2}\right)\left(1+\left|X_{t}\right|^{q}\right)$ and

$$
\mathbb{E}_{\theta^{*}}\left[\left|\int_{0}^{t} f\left(X_{s}, \theta\right) d s\right|\right] \leq \int_{0}^{t} \mathbb{E}_{\theta^{*}}\left[\left|f\left(X_{s}, \theta\right)\right|\right] d s \leq C_{\theta} \int_{0}^{t} \mathbb{E}_{\theta^{*}}\left[1+\left|X_{s}\right|^{q}\right] d s<\infty,
$$

where the last inequality follows because $X_{t}$ has a finite $q$-moment, see Lemma 2.8 .

Hence, to show that the process $F\left(X_{t}, \theta\right)+\int_{0}^{t} f\left(X_{s}, \theta\right) d s$ is an $\mathbb{F}$-martingale, let $t \geq u \geq 0$ and write

$$
\begin{aligned}
\mathbb{E}_{\theta^{*}} & {\left[F\left(X_{t}, \theta\right)+\int_{0}^{t} f\left(X_{s}, \theta\right) d s \mid \mathcal{F}_{u}\right] } \\
= & \mathbb{E}_{\theta^{*}}\left[\left[\int_{0}^{\infty} \mathbb{E}_{x, \theta^{*}} f\left(X_{t+s}, \theta\right) d s\right]+\int_{0}^{t} f\left(X_{s}, \theta\right) d s \mid \mathcal{F}_{u}\right] \quad \text { (Definition in (2.11)) } \\
= & \mathbb{E}_{\theta^{*}}\left[\int_{0}^{\infty} \mathbb{E}_{X_{t}, \theta^{*}}\left[f\left(X_{s+t}, \theta\right) \mid \mathcal{F}_{t}\right] d s+\int_{0}^{t} f\left(X_{s}, \theta\right) d s \mid \mathcal{F}_{u}\right] \quad \text { (Markov property of } X \text { ) } \\
= & \int_{0}^{u} f\left(X_{s}, \theta\right) d s+\mathbb{E}_{X_{u}, \theta^{*}}\left[\int_{0}^{\infty} f\left(X_{s+u}, \theta\right) d s \mid \mathcal{F}_{u}\right] \\
= & \int_{0}^{u} f\left(X_{s}, \theta\right) d s+F\left(X_{u}, \theta\right),
\end{aligned}
$$

where the second to last equation follows from conditional expectation.

Remark 2.10. Note that in Theorem 2.9 above, we do not require any assumptions on the existence or the regularity of the density function of the process $X$. The regularity of the density function of the jump-diffusion process is difficult to analyse.

The following lemma is useful in the next subsection; see Section 4 in [2] for a proof.

Lemma 2.11. For any $p>0$, there is a positive constant $C_{p}$, such that

$$
\int_{\mathbb{R}}\left(1+|x|^{p}\right) \pi^{\theta^{*}}(d x) \leq C_{p}
$$

In addition, we have that $\left|\nabla_{\theta} \bar{g}(\theta)\right| \leq C_{q} K(1+|\theta|)$ and $\left|\nabla_{\theta}^{2} \bar{g}(\theta)\right| \leq C_{q} K$, where $K$ is the same positive constant as that in Condition 2.5. 


\subsection{Convergence of the deviation term}

In this subsection, we propose a novel method to prove the convergence of the deviation term $\Gamma_{k, \gamma}$, see (2.2) and (2.3). When the process $X$ is a diffusion without jumps, one of the steps to prove the convergence of the SGDCT estimator is to show that the function

$$
G(x, \theta):=\int_{0}^{\infty} \mathbb{E}_{x, \theta^{*}}\left[\nabla_{\theta} g\left(X_{t}, \theta\right)-\nabla_{\theta} \bar{g}(\theta)\right] d t
$$

is a classical solution of the Poisson equation (2.12) when $f(x, \theta)=\nabla_{\theta} g(x, \theta)-\nabla_{\theta} \bar{g}(\theta)$, see Pardoux and Veretennikov [14]. Thus, in the pure-diffusion case, one can apply Itô's lemma to the function $\beta_{t} G$ to obtain the following decomposition

$$
\beta_{\bar{\tau}_{k, \gamma}} G\left(X_{\bar{\tau}_{k, \gamma}}, \theta_{\bar{\tau}_{k, \gamma}}\right)-\beta_{\underline{\tau}_{k}} G\left(X_{\underline{\tau}_{k}}, \theta_{\underline{\tau}_{k}}\right)=\Gamma_{k, \gamma}+\text { martingale + Riemann integral, }
$$

where the deviation term $\Gamma_{k, \gamma}$ is as in (2.3). With this decomposition, one shows that the deviation term $\Gamma_{k, \gamma} \rightarrow 0$ as $k \rightarrow \infty$, by proving that the martingale term, the Riemann integral, and the function $\beta_{t} G\left(X_{t}, \theta_{t}\right)$ converge to zero as $k \rightarrow \infty$.

However, when the process $X$ follows a jump-diffusion, the Poisson equation (2.12) has a non-local term, and to the best of our knowledge there is no general result to show that the Poisson equation with a non-local operator admits a classical solution. It is difficult, or perhaps not possible, to show that the function $G$ in (2.14) is second order differentiable with respect to $x$ when $X$ is a jump-diffusion process. Several papers in the literature also encounter a similar difficulty. Uehara [21] states that the properties of the function $G$ are difficult to determine and may require Malliavin calculus to study the gradient estimation of the function $G$ - the author assumes a stochastic process similar to ours. Moreover, [22] mention that there is no analogue to the results in Pardoux and Veretennikov [15] when the process $X$ has a jump component.

Therefore, in our setup we face the problem that the function $G$ is not differentiable with respect to the variable $x$ because the process $X$ is a jump-diffusion. Hence, we cannot employ the classical Itô formula on the function $G$, so below we prove an 'extended Itô formula' that we employ in our analysis. We provide a brief summary of the steps we follow.

First, we show that $G$ is a locally Lipschitz function with the following bound

$$
|G(x, \theta)-G(y, \theta)| \leq C(1+|\theta|)|x-y|\left(1+|x|^{q}+|y|^{q}\right),
$$

where $C$ is a positive real number that does not depend on $x, y, \theta, q>0$. Then, because $G$ is a weak solution of a Poisson equation, see (2.12), and because it is twice differentiable with respect to $\theta$, we show that

$$
\begin{aligned}
& \beta_{\bar{\tau}_{k, \gamma}} G\left(X_{\bar{\tau}_{k, \gamma}}, \theta_{\bar{\tau}_{k, \gamma}}\right)-\beta_{\underline{\tau}_{k}} G\left(X_{\underline{\tau}_{k}}, \theta_{\underline{\tau}_{k}}\right) \\
& \quad=\Gamma_{k, \gamma}+\text { martingale + Riemann integral + covariation, }
\end{aligned}
$$

where the covariation term in the equation above is a càdlàg process.

Second, we study the terms in the above equation in the limit $k \rightarrow \infty$. We show that the left-hand side of (2.16) converges to zero and each of the last three terms on the right-hand side of (2.16) also converges to zero. Therefore, the representation in (2.16) implies that the deviation term $\Gamma_{k, \gamma} \rightarrow 0$ as $k \rightarrow \infty$.

Now, we state a lemma and then prove that the function $G$ is locally Lipschitz. The lemma uses the result that the function $\mathbb{E}_{x, \theta^{*}}\left[f\left(X_{t}, \theta\right)\right]$ is locally Lipschitz with respect to $x$, see Theorem 2.2 in Wang [23]. 
Lemma 2.12. Suppose Conditions 2.1 to 2.5 hold and let $C>0, q>0$. Assume a function $f: \mathbb{R}^{n+d} \rightarrow$ $\mathbb{R}^{d}$ satisfies $|f(x, \theta)-f(y, \theta)| \leq C\left(1+|x|^{q}+|y|^{q}\right)|x-y|$ for all $x, y$ and $t \geq 0$. Then there exist constants $\delta \in(0,1)$ and $C_{\delta}>0$, such that for all $\theta \in \mathbb{R}^{d}$ the following inequality holds

$$
\begin{aligned}
& \left|\mathbb{E}_{x, \theta^{*}}\left[f\left(X_{t}, \theta\right)\right]-\mathbb{E}_{y, \theta^{*}}\left[f\left(X_{t}, \theta\right)\right]\right| \\
& \quad \leq \begin{cases}\left(2+\delta^{-1}\right) e^{-C_{\delta} t} C\left(1+|x|^{q}+|y|^{q}\right)|x-y|, & |x-y| \leq \delta, \\
2 \delta^{-1} C\left(1+|x|^{q}+|y|^{q}\right)|x-y|, & |x-y|>\delta,\end{cases}
\end{aligned}
$$

where the constant $C$ does not depend on $f$.

For a proof see Section 4 in the online supplement [2].

Next, we show that the function $G$ in (2.14) is locally Lipschitz in the variable $x$. We employ Conditions 2.1 to 2.6 , which are easily checked, to show the dissipativity (stability with respect to initial condition) of the process $X$. The main idea of the proof of the proposition below is that for a small value of $t$, we use Lemma 2.12 to control the value $\left|\mathbb{E}_{x, \theta^{*}}\left[\tilde{g}\left(X_{t}, \theta\right)\right]-\mathbb{E}_{y, \theta^{*}}\left[\tilde{g}\left(X_{t}, \theta\right)\right]\right|$, where $\tilde{g}(x, \theta):=\nabla_{\theta} g(x, \theta)-\nabla_{\theta} \bar{g}(\theta)$. And for a large value of $t$, we use the exponential ergodic property of the process $X$. These two results imply the following proposition.

Proposition 2.13. Suppose Conditions 2.1 to 2.5 hold and let $q>0$. Then, the function $G$ in (2.14) is locally Lipschitz, with respect to $x$ and it is twice-differentiable with respect to $\theta$. Thus, there exists a positive constant $C_{G, q}$, such that

$$
|G(x, \theta)-G(y, \theta)| \leq C_{G, q}(1+|\theta|)|x-y|\left(1+|x|^{q}+|y|^{q}\right)
$$

and

$$
\sum_{i=1}^{2}\left|\frac{\partial^{i} G}{\partial \theta^{i}}(x, \theta)\right| \leq C_{G, q}\left(1+|x|^{q}\right),
$$

for all $x, y, \theta$.

Proof. Let $\tilde{g}(x, \theta):=\nabla_{\theta} g(x, \theta)-\nabla_{\theta} \bar{g}(\theta)$. Then, due to Condition 2.5, write

$$
|\tilde{g}(x, \theta)-\tilde{g}(y, \theta)|=\left|\nabla_{\theta} g(x, \theta)-\nabla_{\theta} g(y, \theta)\right| \leq K(1+|\theta|)|x-y|\left(1+|x|^{q}+|y|^{q}\right) .
$$

Let $\delta$ be the same constant as that in Lemma 2.12. Then, for $|x-y| \leq \delta$, we have

$$
\begin{aligned}
|G(x, \theta)-G(y, \theta)| & \leq \int_{0}^{\infty}\left|\mathbb{E}_{x, \theta^{*}}\left[\tilde{g}\left(X_{t}, \theta\right)\right]-\mathbb{E}_{y, \theta^{*}}\left[\tilde{g}\left(X_{t}, \theta\right)\right]\right| d t \\
& \leq \int_{0}^{\infty}\left(2+\delta^{-1}\right) e^{-C_{\delta} t} K(1+|\theta|)\left(1+|x|^{q}+|y|^{q}\right)|x-y| d t \\
& \leq \frac{2+\delta^{-1}}{C_{\delta}} K(1+|\theta|)\left(1+|x|^{q}+|y|^{q}\right)|x-y|,
\end{aligned}
$$

where the second inequality follows from Lemma 2.12. Now, consider the case $|x-y| \geq \delta$ and recall that

$$
\left\|\mathbb{P}_{t}(x, \cdot)-\pi^{\theta^{*}}(\cdot)\right\|_{1+|x|^{q}} \leq C_{1}\left(1+|x|^{q}\right) e^{-C_{2} t}
$$


from Lemma 2.8. Use the triangle inequality to write

$$
\left\|\mathbb{P}_{t}(x, \cdot)-\mathbb{P}_{t}(y, \cdot)\right\|_{1+|x|^{q}} \leq 2 C_{1}\left(1+|x|^{q}+|y|^{q}\right) e^{-C_{2} t} .
$$

Therefore, for $t_{0} \geq\left(1 / C_{2}\right) \log \left(C_{1} /\left(C_{2} \delta\right)\right)$, we have

$$
\begin{aligned}
\int_{t_{0}}^{\infty}\left|\mathbb{E}_{x, \theta^{*}}\left[\tilde{g}\left(X_{t}, \theta\right)\right]-\mathbb{E}_{y, \theta^{*}}\left[\tilde{g}\left(X_{t}, \theta\right)\right]\right| d t & \leq K(1+|\theta|) \int_{t_{0}}^{\infty} 2 C_{1}\left(1+|x|^{q}+|y|^{q}\right) e^{-C_{2} t} d t \\
& =2 K(1+|\theta|)\left(C_{1} / C_{2}\right)\left(1+|x|^{q}+|y|^{q}\right) e^{-C_{2} t_{0}} \\
& \leq 2 K(1+|\theta|)\left(1+|x|^{q}+|y|^{q}\right)|x-y|,
\end{aligned}
$$

where the last inequality holds because $t_{0} \geq\left(1 / C_{2}\right) \log \left(C_{1} /\left(C_{2} \delta\right)\right)$. From Lemma 2.12, write

$$
\begin{aligned}
& \int_{0}^{t_{0}}\left|\mathbb{E}_{x, \theta^{*}}\left[\tilde{g}\left(X_{t}, \theta\right)\right]-\mathbb{E}_{y, \theta^{*}}\left[\tilde{g}\left(X_{t}, \theta\right)\right]\right| d t \\
& \quad \leq K(1+|\theta|) \int_{0}^{t_{0}} \frac{2 C_{\delta}}{\delta}\left(1+|x|^{q}+|y|^{q}\right)|x-y| d t \\
& \quad \leq K(1+|\theta|) \tilde{C}_{\delta}\left(1+|x|^{q}+|y|^{q}\right)|x-y|,
\end{aligned}
$$

where $\tilde{C}_{\delta}$ is a constant. Therefore, (2.17) holds by (2.20), (2.21), (2.22). Note that we can interchange the differentiation with the integration operator, therefore

$$
\left|\frac{\partial^{i} G}{\partial \theta^{i}}(x, \theta)\right| \leq \int_{0}^{\infty}\left|\mathbb{E}_{x, \theta^{*}}\left[\frac{\partial^{i} \nabla_{\theta} g}{\partial \theta^{i}}\left(X_{t}, \theta\right)-\frac{\partial^{i} \nabla_{\theta} \bar{g}}{\partial \theta^{i}}(\theta)\right]\right| d t \leq C_{q}\left(1+|x|^{q}\right),
$$

where the last inequality follows from Lemma 2.11 .

In the next lemma, we construct predictable representation processes $\tilde{G}_{1}$ and $\tilde{G}_{2}$ for the process $G\left(X_{t}, \theta\right)+\int_{0}^{t}\left(\nabla_{\theta} g\left(X_{s}, \theta\right)-\nabla_{\theta} \bar{g}(\theta)\right) d s$, which are also measurable with respect to the variable $\theta$.

Lemma 2.14 (Martingale representation ). For each $\theta$, let

$$
M_{t}^{\theta}:=G\left(X_{t}, \theta\right)-G\left(X_{0}, \theta\right)+\int_{0}^{t}\left(\nabla_{\theta} g\left(X_{s}, \theta\right)-\nabla_{\theta} \bar{g}(\theta)\right) d s,
$$

which is a martingale under $\mathbb{P}_{\theta^{*}}$, see Theorem 2.9 above. Then, there exist measurable functions $\tilde{G}_{1}(x, \theta)$ and $\tilde{G}_{2}(x, \theta, z)$ that are continuous with respect to $\theta$, such that

$$
M_{t}^{\theta}=\int_{0}^{t} \tilde{G}_{1}\left(X_{s}, \theta\right) d B_{s}+\int_{0}^{t} \int_{\mathbb{R}^{r}} \tilde{G}_{2}\left(X_{s}, \theta, z\right) \tilde{\mu}(d s, d z) .
$$

Recall that $\tilde{\mu}$ is the compensated random measure of the jump component in the process $X$, see (1.2). Thus, there exists a constant $C_{M, q}>0$ independent of $x$ and $\theta$, such that for all $\theta \in \mathbb{R}^{d}$, the following two inequalities hold:

$$
\begin{aligned}
\left|\tilde{G}_{1}(x, \theta)\right| & \leq C_{M, q}(1+|\theta|)\left(1+|x|^{q}\right) \quad \text { and } \\
\left|\tilde{G}_{2}(x, \theta, z)\right| & \leq C_{M, q}(1+|\theta|)|x||z|\left(1+|x|^{q}|z|^{q}+|x|^{q}\right)
\end{aligned}
$$

for all $x$ almost everywhere. 
Proof. First, we show that there exists a Borel measurable function $\tilde{G}(x, \theta)$ that agrees with the weak derivative of $G$ in $x$ almost everywhere and that it is continuous with respect to $\theta$. Let

$$
\hat{G}_{i}(x, \theta):=\lim \sup _{h \rightarrow 0}\left\{\frac{\nabla_{\theta} G\left(x+h e_{i}, \theta\right)-\nabla_{\theta} G(x, \theta)}{h}\right\},
$$

where $e_{i}$ is the unit vector (the vector whose $i$ th entry is 1 and 0 elsewhere) and the function $\hat{G}_{i}$ takes values in $\mathbb{R}^{d} \times \mathbb{R}^{d}$, which is a Borel measurable function and agrees with the weak derivative of $\nabla_{\theta} G$ with respect to $x$ in the $i$ th coordinate almost everywhere. Therefore, we have that

$$
\int \phi \hat{G}_{i}(x, \theta) d x=-\int \phi_{i} \nabla_{\theta} G(x, \theta) d x
$$

for all $1 \leq i \leq n$ and $\phi \in C_{c}^{\infty}\left(\mathbb{R}^{n}\right)$ where $\phi_{i}$ is the derivative of the $i$ th coordinate of the function $\phi$.

Next, define the function

$$
\tilde{G}(x, \theta):=\tilde{G}(x, 0)+\int_{0}^{1}\left[\hat{G}_{1}(x, \theta t) \theta, \ldots, \hat{G}_{n}(x, \theta t) \theta\right] d t,
$$

where $\tilde{G}=\left[\tilde{G}_{1}, \ldots, \tilde{G}_{n}\right]$ takes values in $\mathbb{R}^{d} \times \mathbb{R}^{n}$ and

$$
\tilde{G}_{i}(x, 0):=\lim \sup _{h \rightarrow 0}\left\{\frac{G\left(x+h e_{i}, 0\right)-G(x, 0)}{h}\right\} .
$$

Therefore, we have that

$$
\int \phi \tilde{G}_{i}(x, 0) d x=-\int \phi_{i} G(x, 0) d x,
$$

because the function $\tilde{G}_{i}(x, 0)$ agrees with the weak derivative of the function $G(x, 0)$ almost everywhere. For all $\theta$ and $\phi \in C_{c}^{\infty}\left(\mathbb{R}^{n}\right)$, by the definition of $\tilde{G}$ and Fubini's theorem, write

$$
\begin{aligned}
\int \phi \tilde{G}_{k}(x, \theta) d x & =\int \phi\left(\tilde{G}_{k}(x, 0)+\int_{0}^{1} \hat{G}_{k}(x, \theta t) \theta d t\right) d x \\
& =\int \phi \tilde{G}_{k}(x, 0) d x+\iint_{0}^{1} \phi \hat{G}_{k}(x, \theta t) \theta d t d x \\
& =-\int \phi_{k} G(x, 0) d x+\int_{0}^{1} \int \phi \hat{G}_{k}(x, \theta t) \theta d x d t \\
& =-\int \phi_{k} G(x, 0) d x+\int_{0}^{1}-\int \phi_{k} \nabla_{\theta} G(x, \theta t) \theta d x d t \\
& =-\int \phi_{k} G(x, 0) d x-\int \phi_{k} \int_{0}^{1} \nabla_{\theta} G(x, \theta t) \theta d t d x \\
& =-\int \phi_{k} G(x, 0) d x-\int \phi_{k}(G(x, \theta)-G(x, 0)) d t d x \\
& =-\int \phi_{k} G(x, \theta) d x,
\end{aligned}
$$


where the third equation follows from (2.28), the fourth equation follows from (2.27). Therefore, from (2.29), for each $\theta$, the function $\tilde{G}(x, \theta)$ agrees with the weak derivative in $x$ of the function $G(x, \theta)$ almost everywhere.

The function $\tilde{G}$ is continuous with respect to the variable $\theta$ and Borel measurable with respect to $x$. Therefore, by the Carathéodory theorem, $\tilde{G}(x, \theta)$ is a Borel measurable function continuous in $\theta$ and it agrees with the weak derivative of $G$ in $x$ almost everywhere.

Next, we show that

$$
[G(X, \theta), B]_{t}=\int_{0}^{t} \tilde{G}\left(X_{s}, \theta\right) d[X, B]_{s},
$$

where $[G(X, \theta), B]$ denotes the covariation of the processes $G(X, \theta)$ and $B$.

If the function $G$ is differentiable with respect to $x$, then it is straightforward to show that (2.30) holds. However, we need to analyse the function $G$ very carefully because it is only locally Lipschitz. Let $C_{\text {loc }}\left(\mathbb{R}^{d}\right)$ be the space of locally Lipschitz functions and let $\mathbb{D}$ be the space of càdlàg processes equipped with the metric of uniform convergence in probability (u.c.p.) topology. Then the mapping

$$
G(\cdot, \theta) \mapsto[G(X, \theta), B]
$$

is a continuous mapping from $C_{\mathrm{loc}}\left(\mathbb{R}^{d}\right)$ to $\mathbb{D}$ by a similar argument to that in the proof of Theorem 3.8 in Errami et al. [6]. Consider $G^{k}=G * \phi_{k}$ where $*$ is the convolution operator and $\left\{\phi_{k}\right\}_{k \geq 1}$ is a sequence of classical mollifiers converging to the Dirac measure at 0 . Then,

$$
\left[G^{k}(X, \theta), B\right]_{t}=\int_{0}^{t} \nabla_{x} G^{k}\left(X_{s}, \theta\right) d[X, B]_{s},
$$

because the function $G^{k}$ is differentiable with respect to $x$. Moreover, the continuity of the mapping in (2.31) implies that

$$
\begin{aligned}
{[G(X, \theta), B]_{t} } & =\lim _{k \rightarrow \infty}\left[G^{k}(X, \theta), B\right]_{t} \\
& =\lim _{k \rightarrow \infty} \int_{0}^{t} \nabla_{x} G^{k}\left(X_{s}, \theta\right) d[X, B]_{s}=\int_{0}^{t} \tilde{G}\left(X_{s}, \theta\right) d[X, B]_{s},
\end{aligned}
$$

$\mathbb{P}_{\theta^{*}}$ almost surely, where the last equation follows from the dominated convergence argument - see Lemma 4.3 in the online supplement [2] for a proof.

This shows (2.30), so we write

$$
[G(X, \theta), B]_{t}=\int_{0}^{t} \tilde{G}_{1}\left(X_{s}, \theta\right) d s,
$$

where $\tilde{G}_{1}(x, \theta):=\tilde{G}(x, \theta) \sigma(x)$. Therefore, from the boundedness of the volatility term in the process $X$, the function $\tilde{G}_{1}$ satisfies (2.25) for all $x$ almost everywhere by (2.17) and (2.26).

Next, consider the jump part of the process $M_{t}^{\theta}$. From the definition of covariation, write

$$
\begin{aligned}
{[G(X, \theta), Z]_{t} } & =\lim _{\left\|P_{n}\right\| \rightarrow 0} \sum_{P_{n}}\left(G\left(X_{t_{i+1}}, \theta\right)-G\left(X_{t_{i}}, \theta\right)\right)\left(Z_{t_{i+1}}-Z_{t_{i}}\right) \\
& =\int_{0}^{t} \int_{\mathbb{R}^{r}}\left[G\left(X_{s^{-}}+\xi\left(X_{s^{-}}, z\right), \theta\right)-G\left(X_{s^{-}}, \theta\right)\right] \tilde{\mu}(d s, d z) \\
& =\int_{0}^{t} \int_{\mathbb{R}^{r}} \tilde{G}_{2}\left(X_{s^{-}}, \theta, z\right) \tilde{\mu}(d s, d z),
\end{aligned}
$$


where $P_{n}$ is a partition of the interval $[0, t]$ and $\tilde{G}_{2}(x, \theta, z):=G(x+\xi(x, z), \theta)-G(x, \theta)$. From (2.17), write

$$
\begin{aligned}
\left|\tilde{G}_{2}(x, \theta, z)\right| & =|G(x+\xi(x, z), \theta)-G(x, \theta)| \\
& \leq C_{G, q}(1+|\theta|)|\xi(x, z)|\left(1+|x+\xi(x, z)|^{q}+|x|^{q}\right) \\
& \leq C_{M, q}(1+|\theta|)|x||z|\left(1+|x|^{q}|z|^{q}+|x|^{q}\right),
\end{aligned}
$$

where the last inequality follows from Condition 2.3.

We note that the existence of the martingale representation of the process $M^{\theta}$ is a result of Theorem 1.1 in Kunita [9].

Now, we show that the difference $G\left(X_{t}, \theta_{t}\right)-G\left(X_{0}, \theta_{0}\right)$ is the sum of a stochastic integral, a Riemann integral, and a covariation term. Our approach is based on Föllmer et al. [7].

Proposition 2.15 (Extended Itô lemma). Suppose Conditions 2.1 to 2.5 hold. Let the function $G$ be as in (2.14) and let the functions $\tilde{G}_{1}$ and $\tilde{G}_{2}$ be as in Lemma 2.14. The following extended Itô lemma holds:

$$
\begin{aligned}
G\left(X_{t}, \theta_{t}\right)= & G\left(X_{0}, \theta_{0}\right)+\int_{0}^{t} \mathcal{A}_{x, \theta^{*}} G\left(X_{s}, \theta_{s}\right) d s+\int_{0}^{t} \tilde{G}_{1}\left(X_{s}, \theta_{s}\right) d B_{s} \\
& +\int_{0}^{t} \int_{\mathbb{R}} \tilde{G}_{2}\left(X_{s}, \theta_{s}, z\right) \tilde{\mu}(d s, d z)+\int_{0}^{t} \nabla_{\theta} G\left(X_{s}, \theta_{s}\right) d \theta_{s} \\
& +\left[\int_{0}^{t} \frac{1}{2} \nabla_{\theta}^{2} G_{k}\left(X_{s}, \theta_{s}\right) d[\theta, \theta]_{s}\right]_{k=1}^{d}+\left[\nabla_{\theta} G(X, \theta), \theta\right]_{t}^{x},
\end{aligned}
$$

where $G_{k}$ is a real-valued function for all $1 \leq k \leq d, G=\left[G_{1}, \ldots, G_{d}\right]^{\top}$, and the covariation with respect to $x,\left[\nabla_{\theta} G(X, \theta), \theta\right]_{t}^{x}$, is defined as

$$
\left[\nabla_{\theta} G(X, \theta), \theta\right]_{t}^{x}:=\lim _{\left\|P_{n}\right\| \rightarrow 0} \sum_{P_{n}}\left(\nabla_{\theta} G\left(X_{t_{i+1}}, \theta_{t_{i}}\right)-\nabla_{\theta} G\left(X_{t_{i}}, \theta_{t_{i}}\right)\right)\left(\theta_{t_{i+1}}-\theta_{t_{i}}\right),
$$

and recall that $\mathcal{A}_{x, \theta^{*}}$ is the extended generator of the process $X$, see (2.12).

Proof. Let $\left\{P_{n}\right\}_{n \geq 1}$ be a sequence of partitions of the interval $[0, t]$, such that the maximal mesh size of $P_{n}$ converges to zero as $n$ goes to infinity. For a partition $P_{n}$, write

$$
\begin{aligned}
G & \left(X_{t}, \theta_{t}\right)-G\left(X_{0}, \theta_{0}\right) \\
& =\sum_{P_{n}}\left\{G\left(X_{t_{i+1}}, \theta_{t_{i+1}}\right)-G\left(X_{t_{i}}, \theta_{t_{i}}\right)\right\} \\
& =\sum_{P_{n}}\left\{G\left(X_{t_{i+1}}, \theta_{t_{i+1}}\right)-G\left(X_{t_{i+1}}, \theta_{t_{i}}\right)+G\left(X_{t_{i+1}}, \theta_{t_{i}}\right)-G\left(X_{t_{i}}, \theta_{t_{i}}\right)\right\} .
\end{aligned}
$$

Consider each component on the right-hand of (2.36) individually and because the function $G$ is twice differentiable with respect to $\theta$, use Taylor's expansion to write

$$
\begin{aligned}
& G\left(X_{t_{i+1}}, \theta_{t_{i+1}}\right)-G\left(X_{t_{i+1}}, \theta_{t_{i}}\right) \\
& \quad=\nabla_{\theta} G\left(X_{t_{i+1}}, \theta_{t_{i}}\right)\left(\theta_{t_{i+1}}-\theta_{t_{i}}\right)
\end{aligned}
$$




$$
\begin{aligned}
& +\left[\frac{1}{2} \operatorname{Tr}\left(\left(\theta_{t_{i+1}}-\theta_{t_{i}}\right)^{\top} \nabla_{\theta}^{2} G_{k}\left(X_{t_{i+1}}, \theta_{t_{i}}\right)\left(\theta_{t_{i+1}}-\theta_{t_{i}}\right)\right)\right]_{k=1}^{d}+R\left(X_{t_{i+1}}, \theta_{t_{i}}\right) \\
= & \nabla_{\theta} G\left(X_{t_{i}}, \theta_{t_{i}}\right)\left(\theta_{t_{i+1}}-\theta_{t_{i}}\right) \\
& +\left[\frac{1}{2} \operatorname{Tr}\left(\left(\theta_{t_{i+1}}-\theta_{t_{i}}\right)^{\top} \nabla_{\theta}^{2} G_{k}\left(X_{t_{i}}, \theta_{t_{i}}\right)\left(\theta_{t_{i+1}}-\theta_{t_{i}}\right)\right)\right]_{k=1}^{d} \\
& +\left(\nabla_{\theta} G\left(X_{t_{i+1}}, \theta_{t_{i}}\right)-\nabla_{\theta} G\left(X_{t_{i}}, \theta_{t_{i}}\right)\right)\left(\theta_{t_{i+1}}-\theta_{t_{i}}\right) \\
& +\left[\frac{1}{2} \operatorname{Tr}\left(\left(\theta_{t_{i+1}}-\theta_{t_{i}}\right)^{\top}\left(\nabla_{\theta}^{2} G\left(X_{t_{i+1}}, \theta_{t_{i}}\right)-\nabla_{\theta}^{2} G\left(X_{t_{i}}, \theta_{t_{i}}\right)\right)\left(\theta_{t_{i+1}}-\theta_{t_{i}}\right)\right)\right]_{k=1}^{d} \\
& +R\left(X_{t_{i+1}}, \theta_{t_{i}}\right),
\end{aligned}
$$

where $R$ is the remainder function and the operation $\operatorname{Tr}[\cdot]$ denotes the trace of a matrix. Then, by standard stochastic integration and Riemann integration, the following equations hold:

$$
\begin{aligned}
\lim _{\left\|P_{n}\right\| \rightarrow 0} \sum_{P_{n}} \nabla_{\theta} G\left(X_{t_{i}}, \theta_{t_{i}}\right)\left(\theta_{t_{i+1}}-\theta_{t_{i}}\right) & =\int_{0}^{t} \nabla_{\theta} G\left(X_{s}, \theta_{s}\right) d \theta_{s}, \\
\lim _{\left\|P_{n}\right\| \rightarrow 0} \sum_{P_{n}} \frac{1}{2} \operatorname{Tr}\left(\left(\theta_{t_{i+1}}-\theta_{t_{i}}\right)^{\top} \nabla_{\theta}^{2} G_{k}\left(X_{t_{i}}, \theta_{t_{i}}\right)\left(\theta_{t_{i+1}}-\theta_{t_{i}}\right)\right) & =\int_{0}^{t} \frac{1}{2} \nabla_{\theta}^{2} G_{k}\left(X_{s}, \theta_{s}\right) d[\theta, \theta]_{s},
\end{aligned}
$$

for all $1 \leq k \leq d$, because $\nabla_{\theta} G$ and $\nabla_{\theta}^{2} G$ are continuous with respect to $\theta$. Moreover, we have that

$$
\lim _{\left\|P_{n}\right\| \rightarrow 0} \sum_{P_{n}} R\left(X_{t_{i+1}}, \theta_{t_{i}}\right)=0
$$

because the remainder function $R$ depends on the third order of the increment of the process $\theta$ and the quadratic variation of the process $\theta$ is finite.

The locally Lipschitz property of the function $G$ implies that for all $1 \leq k \leq d$

$$
\begin{aligned}
& \left|\nabla_{\theta}^{2} G_{k}\left(X_{t_{i+1}}, \theta_{t_{i}}\right)-\nabla_{\theta}^{2} G_{k}\left(X_{t_{i}}, \theta_{t_{i}}\right)\right| \\
& \quad \leq C_{G, q}\left(1+\left|X_{t_{i+1}}\right|^{q}+\left|X_{t_{i}}\right|^{q}\right)\left|X_{t_{i+1}}-X_{t_{i}}\right| \\
& \quad \leq 2 C_{G, q}\left(1+\sup _{0 \leq s \leq t}\left|X_{s}\right|^{q}\right)\left(\sum_{t_{i}, t_{i+1}}|\Delta X|+\left|X_{t_{i+1}}^{c}-X_{t_{i}}^{c}\right|\right),
\end{aligned}
$$

where $X^{c}$ is a continuous component of the process $X$ and $\sum_{t_{i}, t_{i+1}}|\Delta X|$ is the sum of all jumps in the interval $\left[t_{i}, t_{i+1}\right]$. The following sum converges

$$
\lim _{\left\|P_{n}\right\| \rightarrow 0} \sum_{P_{n}}\left(\theta_{t_{i+1}}-\theta_{t_{i}}\right)^{\top}\left|X_{t_{i+1}}^{c}-X_{t_{i}}^{c}\right|\left(\theta_{t_{i+1}}-\theta_{t_{i}}\right)=0
$$

because the process $X^{c}$ is continuous and the quadratic variation of the process $\theta$ is finite. We denote by $J$ the sum of the jumps of the process $X$ in $[0, T]$. Then, the random variable $J$ is finite with probability one because $v$ is a finite measure. Therefore,

$$
\lim _{\left\|P_{n}\right\| \rightarrow 0} \sum_{P_{n}} \sum_{t_{i}, t_{i+1}}\left(\theta_{t_{i+1}}-\theta_{t_{i}}\right)^{\top}|\Delta X|\left(\theta_{t_{i+1}}-\theta_{t_{i}}\right) \leq J^{2} \lim _{\left\|P_{n}\right\| \rightarrow 0} \sup _{i}\left|\theta_{t_{i+1}}-\theta_{t_{i}}\right|^{2}=0 .
$$


The last equation holds because the process $\theta$ is continuous and its quadratic variation is finite. From (2.39), (2.40), (2.41), we have that

$$
\lim _{\left\|P_{n}\right\| \rightarrow 0} \sum_{P_{n}} \frac{1}{2} \operatorname{Tr}\left(\left(\theta_{t_{i+1}}-\theta_{t_{i}}\right)^{\top}\left(\nabla_{\theta}^{2} G_{k}\left(X_{t_{i+1}}, \theta_{t_{i}}\right)-\nabla_{\theta}^{2} G_{k}\left(X_{t_{i}}, \theta_{t_{i}}\right)\right)\left(\theta_{t_{i+1}}-\theta_{t_{i}}\right)\right)=0 .
$$

Next, we show that the covariation term is well-defined. Recall that (see (2.37))

$$
\lim _{\left\|P_{n}\right\| \rightarrow 0} \sum_{P_{n}} \nabla_{\theta} G\left(X_{t_{i}}, \theta_{t_{i}}\right)\left(\theta_{t_{i+1}}-\theta_{t_{i}}\right)=\int_{0}^{t} \nabla_{\theta} G\left(X_{s}, \theta_{s}\right) d \theta_{s},
$$

with convergence in u.c.p because the processes $X$ and $\theta$ are semimartingales and the function $\nabla_{\theta} G(x, \theta)$ is continuous, see Protter [16]. In Lemma 4.2 in the online supplement [2], we show that the coupled process $(X, \theta)$ satisfies the time reversal property, so we write

$$
\begin{aligned}
& \lim _{\left\|P_{n}\right\| \rightarrow 0} \sum \nabla_{\theta} G\left(X_{t_{i+1}}, \theta_{t_{i}}\right)\left(\theta_{t_{i+1}}-\theta_{t_{i}}\right) \\
& =\lim _{\left\|P_{n}\right\| \rightarrow 0}\left\{\sum_{t_{i} \leq t} \nabla_{\theta} G\left(X_{t-t_{i+1}}, \theta_{t-t_{i}}\right)\left(\theta_{t-t_{i+1}}-\theta_{t-t_{i}}\right)\right\} \circ \mathbf{R} \\
& =\lim _{\left\|P_{n}\right\| \rightarrow 0}-\left\{\sum_{s_{i}=t-t_{i} \leq t} \nabla_{\theta} G\left(X_{s_{i}}, \theta_{s_{i+1}}\right)\left(\theta_{s_{i+1}}-\theta_{s_{i}}\right)\right\} \circ \mathbf{R},
\end{aligned}
$$

where $\mathbf{R}$ is the time reversal operator (i.e., $X_{s} \circ \mathbf{R}=X_{t-s}$ ). Therefore, it suffices to show that the limit on the right-hand of (2.43) exists. Consider the sum

$$
\begin{aligned}
& \sum_{s_{i} \leq t} \nabla_{\theta} G\left(X_{s_{i}}, \theta_{s_{i+1}}\right)\left(\theta_{s_{i+1}}-\theta_{s_{i}}\right) \\
& =\sum_{s_{i} \leq t} \nabla_{\theta} G\left(X_{s_{i}}, \theta_{s_{i}}\right)\left(\theta_{s_{i+1}}-\theta_{s_{i}}\right) \\
& \quad+\sum_{s_{i} \leq t}\left[\frac{1}{2} \operatorname{Tr}\left(\left(\theta_{s_{i+1}}-\theta_{s_{i}}\right)^{\top} \nabla_{\theta}^{2} G_{k}\left(X_{s_{i}}, \theta_{s_{i}^{\prime}}\right)\left(\theta_{s_{i+1}}-\theta_{s_{i}}\right)\right)\right]_{k=1}^{d},
\end{aligned}
$$

and note that

$$
\lim _{\left\|P_{n}\right\| \rightarrow 0} \sum_{P_{n}} \nabla_{\theta} G\left(X_{t_{i+1}}, \theta_{t_{i}}\right)\left(\theta_{t_{i+1}}-\theta_{t_{i}}\right)
$$

is well-defined because the limits of both terms on the right-hand side of (2.44) exist; thus, the covariation with respect to $x$ in (2.35) is well-defined. Therefore,

$$
\begin{aligned}
& \lim _{\left\|P_{n}\right\| \rightarrow 0} \sum_{P_{n}}\left(G\left(X_{t_{i+1}}, \theta_{t_{i+1}}\right)-G\left(X_{t_{i+1}}, \theta_{t_{i}}\right)\right) \\
& \quad=\int_{0}^{t} \nabla_{\theta} G\left(X_{s}, \theta_{s}\right) d \theta_{s}+\left[\int_{0}^{t} \frac{1}{2} \nabla_{\theta}^{2} G_{k}\left(X_{s}, \theta_{s}\right) d[\theta, \theta]_{s}\right]_{k=1}^{d}+\left[\nabla_{\theta} G(X, \theta), \theta\right]_{t}^{x},
\end{aligned}
$$


from (2.35), (2.37), (2.42). Now, consider the second component of the sum on the right-hand side of (2.36). From Lemma 2.14, the following equation holds:

$$
\begin{aligned}
G\left(X_{t_{i+1}}, \theta_{t_{i}}\right)-G\left(X_{t_{i}}, \theta_{t_{i}}\right)= & \int_{t_{i}}^{t_{i+1}} \mathcal{A}_{x, \theta^{*}} G\left(X_{s}, \theta_{t_{i}}\right) d s+\int_{t_{i}}^{t_{i+1}} \tilde{G}_{1}\left(X_{s}, \theta_{t_{i}}\right) d B_{s} \\
& +\int_{t_{i}}^{t_{i+1}} \int_{\mathbb{R}} \tilde{G}_{2}\left(X_{s}, \theta_{t_{i}}, z\right) \tilde{\mu}(d s, d z) .
\end{aligned}
$$

From Lemma 2.14, we have that

$$
\left|\tilde{G}_{1}\left(X_{s}, \theta_{u}\right)\right| \leq C_{M, q}\left(1+\left|\theta_{u}\right|\right)\left(1+\left|X_{S}\right|^{q}\right),
$$

on $d \mathbb{P}_{\theta^{*}} \times d t$ almost everywhere. The sequence $\tilde{G}_{1}\left(X_{s}, \theta_{t_{i}}\right)$ converges to $\tilde{G}_{1}\left(X_{s}, \theta_{s}\right)$ pointwise because the function $\tilde{G}_{1}$ is continuous with respect to the variable $\theta$ and because the process $\theta_{s}$ is continuous. Use Lemma 2.8, the Lebesgue's dominated convergence theorem for stochastic integration, and (2.47), to write

$$
\lim _{\left\|P_{n}\right\| \rightarrow 0} \sum_{P_{n}} \int_{t_{i}}^{t_{i+1}} \tilde{G}_{1}\left(X_{s}, \theta_{t_{i}}\right) d B_{s}=\int_{0}^{t} \tilde{G}_{1}\left(X_{s}, \theta_{s}\right) d B_{s} .
$$

For the jump part, we use the same argument as that above to obtain (2.48), and write

$$
\lim _{\left\|P_{n}\right\| \rightarrow 0} \sum_{P_{n}} \int_{t_{i}}^{t_{i+1}} \int_{\mathbb{R}} \tilde{G}_{2}\left(X_{s}, \theta_{t_{i}}, z\right) \tilde{\mu}(d s, d z)=\int_{0}^{t} \int_{\mathbb{R}} \tilde{G}_{2}\left(X_{s}, \theta_{s}, z\right) \tilde{\mu}(d s, d z) .
$$

The last term on the right-hand side of (2.46) is

$$
\int_{t_{i}}^{t_{i+1}} \mathcal{A}_{x, \theta^{*}} G\left(X_{s}, \theta_{t_{i}}\right) d s=\int_{t_{i}}^{t_{i+1}}\left(\nabla_{\theta} g\left(X_{s}, \theta_{t_{i}}\right)-\nabla_{\theta} \bar{g}\left(\theta_{t_{i}}\right)\right) d s .
$$

By the assumptions on the function $g$, we have that

$$
\left|\nabla_{\theta} g(x, \theta)-\nabla_{\theta} \bar{g}(\theta)\right| \leq C(1+|\theta|)\left(1+|x|^{q}\right),
$$

for some constants $C>0$ and $q>0$. Therefore, apply the Lebesgue's dominated convergence theorem to the Riemann integral and write

$$
\begin{aligned}
\lim _{\left\|P_{n}\right\| \rightarrow 0} \sum_{P_{n}} \int_{t_{i}}^{t_{i+1}}\left(\nabla_{\theta} g\left(X_{s}, \theta_{t_{i}}\right)-\nabla_{\theta} \bar{g}\left(\theta_{t_{i}}\right)\right) d s & =\int_{0}^{t}\left(\nabla_{\theta} g\left(X_{s}, \theta_{s}\right)-\nabla_{\theta} \bar{g}\left(\theta_{s}\right)\right) d s \\
& =\int_{0}^{t} \mathcal{A}_{x, \theta^{*}} G\left(X_{s}, \theta_{s}\right) d s .
\end{aligned}
$$

From (2.48), (2.49), (2.50), we have

$$
\begin{aligned}
\lim _{\left\|P_{n}\right\| \rightarrow 0} \sum_{P_{n}}\left\{G\left(X_{t_{i+1}}, \theta_{t_{i}}\right)-G\left(X_{t_{i}}, \theta_{t_{i}}\right)\right\}= & \int_{0}^{t} \mathcal{A}_{x, \theta^{*}} G\left(X_{s}, \theta_{s}\right) d s+\int_{0}^{t} \tilde{G}_{1}\left(X_{s}, \theta_{s}\right) d B_{s} \\
& +\int_{0}^{t} \int_{\mathbb{R}} \tilde{G}_{2}\left(X_{s}, \theta_{s}, z\right) \tilde{\mu}(d s, d z) .
\end{aligned}
$$

Therefore, (2.45) and (2.51) show that the extended Itô formula in (2.34) holds. 
If the function $G$ is twice differentiable with respect to $x$, then the covariation term in (2.35) is a Riemann integral, and the formula in (2.34) coincides with the classical Itô formula. The function $G$ in (2.14) satisfies the weak Poisson equation (2.12) because the function $\nabla_{\theta} g(x, \theta)-\nabla_{\theta} \bar{g}(\theta)$ is centered as required in Theorem 2.9. Then, in the next proposition we apply Proposition 2.13 and the extended Itô lemma to prove the convergence of the deviation term $\Gamma_{k, \gamma}$.

Before proceeding, we define the following increasing stopping times. For an arbitrary $\kappa>0$ and $\lambda=\lambda(\kappa)>0$

$$
\begin{aligned}
& \underline{\tau}_{k}:=\inf \left\{t>\bar{\tau}_{k-1}:\left|\nabla_{\theta} \bar{g}\left(\theta_{t}\right)\right| \geq \kappa\right\}, \\
& \bar{\tau}_{k}:=\sup \left\{t>\underline{\tau}_{k}:\left|\nabla_{\theta} \bar{g}\left(\theta_{\underline{\tau}_{k}}\right)\right| / 2 \leq\left|\nabla_{\theta} \bar{g}\left(\theta_{s}\right)\right| \leq 2\left|\nabla_{\theta} \bar{g}\left(\theta_{\underline{\tau}_{k}}\right)\right|\right. \\
& \left.\quad \text { for all } s \in\left[\underline{\tau}_{k}, t\right] \text { and } \int_{\underline{\tau}_{k}}^{t} \beta_{s} d s \leq \lambda\right\},
\end{aligned}
$$

where $k=1,2, \ldots$ and $\sigma_{0}=0$, and we denote $\bar{\tau}_{k, \gamma}=\bar{\tau}_{k}+\gamma$ for $\gamma>0$. This sequence of stopping times quantifies the period of time for which $\left|\nabla_{\theta} \bar{g}(\theta)\right|$ is small, see Bertsekas and Tsitsiklis [1].

Proposition 2.16 (Convergence of deviation term). Recall that the deviation term is given by

$$
\Gamma_{k, \gamma}=\int_{\underline{\tau}_{k}}^{\bar{\tau}_{k, \gamma}} \beta_{s}\left(\nabla_{\theta} g\left(X_{s}, \theta_{s}\right)-\nabla_{\theta} \bar{g}\left(\theta_{s}\right)\right) d s .
$$

Assume Conditions 2.1 to 2.6 hold and let $X_{0}=x_{0}$. Then, with probability one, we have that

$$
\left|\Gamma_{k, \gamma}\right| \rightarrow 0 \text { as } k \rightarrow \infty
$$

Proof. The idea of this proof is closely related to the proof of Lemma 3.1 in [17]. Recall that the function $\nabla_{\theta} g(x, \theta)-\nabla_{\theta} \bar{g}(\theta)$ is centered and that $G$ is a solution to the weak Poisson equation (2.12) and that it is locally Lipschitz.

Let $\underline{\tau}$ and $\bar{\tau}$ be stopping times, such that $\underline{\tau} \leq \bar{\tau}$. First, we show that

$$
\begin{aligned}
\beta_{\bar{\tau}} G\left(X_{\bar{\tau}}, \theta_{\bar{\tau}}\right)-\beta_{\underline{\tau}} G\left(X_{\underline{\tau}}, \theta_{\underline{\tau}}\right) \\
=\int_{\underline{\tau}}^{\bar{\tau}} \beta_{s}^{\prime} G\left(X_{s}, \theta_{s}\right) d s+\int_{\underline{\tau}}^{\bar{\tau}} \beta_{s} \mathcal{A}_{x, \theta^{*}} G\left(X_{s}, \theta_{s}\right) d s+\int_{\underline{\tau}}^{\bar{\tau}} \beta_{s} \tilde{G}_{1}\left(X_{s}, \theta_{s}\right) d B_{s} \\
\quad+\int_{\underline{\tau}}^{\bar{\tau}} \int_{\mathbb{R}} \beta_{s} \tilde{G}_{2}\left(X_{s}, \theta_{s}, z\right) \tilde{\mu}(d s, d z)+\int_{\underline{\tau}}^{\bar{\tau}} \beta_{s} \nabla_{\theta} G\left(X_{s}, \theta_{s}\right) d \theta_{s} \\
\quad+\left[\int_{\underline{\tau}}^{\bar{\tau}} \frac{1}{2} \beta_{s} \nabla_{\theta}^{2} G_{k}\left(X_{s}, \theta_{s}\right) d[\theta, \theta]_{s}\right]_{k=1}^{d}+\left[\beta \nabla_{\theta} G(X, \theta), \theta\right]_{\underline{\tau}}^{\bar{\tau}, x},
\end{aligned}
$$

where $\beta_{t}^{\prime}$ is the derivative of $\beta_{t}$ with respect to $t$ and the quadratic covariation term

$$
\left[\beta \nabla_{\theta} G(X, \theta), \theta\right]_{\underline{\tau}}^{\bar{\tau}, x}=\lim _{\left\|P_{n}\right\| \rightarrow 0} \sum_{P_{n}}\left(\beta_{t_{i+1}} \nabla_{\theta} G\left(X_{t_{i+1}}, \theta_{t_{i}}\right)-\beta_{t_{i}} \nabla_{\theta} G\left(X_{t_{i}}, \theta_{t_{i}}\right)\right)\left(\theta_{t_{i+1}}-\theta_{t_{i}}\right) .
$$

Here, $P_{n}$ is a partition of the interval $[\underline{\tau}, \bar{\tau}]$, and (2.53) is well-defined due to the time reversal argument in Proposition 2.15. Write

$$
\beta_{\bar{\tau}} G\left(X_{\bar{\tau}}, \theta_{\bar{\tau}}\right)-\beta_{\underline{\tau}} G\left(X_{\underline{\tau}}, \theta_{\underline{\tau}}\right)=A_{1}^{n}+A_{2}^{n},
$$


where $A_{1}^{n}=\sum_{P_{n}} \beta_{t_{i+1}}\left(G\left(X_{t_{i+1}}, \theta_{t_{i+1}}\right)-G\left(X_{t_{i}}, \theta_{t_{i}}\right)\right)$ and $A_{2}^{n}=\left(\beta_{t_{i+1}}-\beta_{t_{i}}\right) G\left(X_{t_{i}}, \theta_{t_{i}}\right)$. Next, consider

$$
\lim _{n \rightarrow \infty} A_{2}^{n}=\lim _{n \rightarrow \infty} \sum_{P_{n}} \beta_{s_{i+1}}^{\prime}\left(t_{i+1}-t_{i}\right) G\left(X_{t_{i}}, \theta_{t_{i}}\right)=\int_{\underline{\tau}}^{\bar{\tau}} \beta_{s}^{\prime} G\left(X_{s}, \theta_{s}\right) d s,
$$

where the second equation holds because $\sum_{P_{n}} \beta_{s_{i+1}}^{\prime} \mathbf{1}_{\left\{s_{i+1} \in\left[t_{i}, t_{i+1}\right)\right\}} G\left(X_{t_{i}}, \theta_{t_{i}}\right)$ converges to $\beta_{s}^{\prime} G\left(X_{s}, \theta_{s}\right)$ almost surely and by the dominated convergence theorem. Now, consider

$$
\begin{aligned}
\lim _{n \rightarrow \infty} A_{1}^{n}= & \lim _{n \rightarrow \infty} \sum_{P_{n}} \beta_{t_{i+1}}\left(G\left(X_{t_{i+1}}, \theta_{t_{i+1}}\right)-G\left(X_{t_{i}}, \theta_{t_{i}}\right)\right) \\
= & \int_{\underline{\tau}}^{\bar{\tau}} \beta_{s} \mathcal{A}_{x, \theta^{*}} G\left(X_{s}, \theta_{s}\right) d s+\int_{\underline{\tau}}^{\bar{\tau}} \beta_{s} \tilde{G}_{1}\left(X_{s}, \theta_{s}\right) d B_{s}+\int_{\underline{\tau}}^{\bar{\tau}} \beta_{s} \nabla_{\theta} G\left(X_{s}, \theta_{s}\right) d \theta_{s} \\
& +\int_{\underline{\tau}}^{\bar{\tau}} \int_{\mathbb{R}} \beta_{s} \tilde{G}_{2}\left(X_{s}, \theta_{s}, z\right) \tilde{\mu}(d s, d z)+\left[\int_{\underline{\tau}}^{\bar{\tau}} \frac{1}{2} \beta_{s} \nabla_{\theta}^{2} G_{k}\left(X_{s}, \theta_{s}\right) d[\theta, \theta]_{s}\right]_{k=1}^{d} \\
& +\lim _{n \rightarrow \infty} \sum_{P_{n}} \beta_{t_{i+1}}\left(\left[\nabla_{\theta} G(X, \theta), \theta\right]_{t_{i+1}}^{x}-\left[\nabla_{\theta} G(X, \theta), \theta\right]_{t_{i}}^{x}\right),
\end{aligned}
$$

where the second equation follows from Proposition 2.15 and from the dominated convergence theorem.

Next, we must show that

$$
\lim _{n \rightarrow \infty} \sum_{P_{n}} \beta_{t_{i+1}}\left(\left[\nabla_{\theta} G(X, \theta), \theta\right]_{t_{i+1}}^{x}-\left[\nabla_{\theta} G(X, \theta), \theta\right]_{t_{i}}^{x}\right)
$$

is well-defined and it is equal to $\left[\beta \nabla_{\theta} G(X, \theta), \theta\right]_{\tau}^{\bar{\tau}, x}$. From the proof of Proposition 2.15, we have that

$$
\begin{aligned}
& \beta_{t_{i+1}}\left(\left[\nabla_{\theta} G(X, \theta), \theta\right]_{t_{i+1}}^{x}-\left[\nabla_{\theta} G(X, \theta), \theta\right]_{t_{i}}^{x}\right) \\
& \quad=\beta_{t_{i+1}}\left[\int_{t_{i}}^{t_{i+1}} \nabla_{\theta} G\left(X_{s}, \theta_{s}\right) d^{*, x} \theta_{s}-\int_{t_{i}}^{t_{i+1}} \nabla_{\theta} G\left(X_{s}, \theta_{s}\right) d \theta_{s}\right],
\end{aligned}
$$

where $\int_{t_{i}}^{t_{i+1}} \nabla_{\theta} G\left(X_{s}, \theta_{s}\right) d^{*, x} \theta_{s}:=\lim \sum_{t_{i}<s_{i}<t_{i+1}} \nabla_{\theta} G\left(X_{s_{i+1}}, \theta_{s_{i}}\right)\left(\theta_{s_{i+1}}-\theta_{s_{i}}\right)$ is a backward integration. Take the limit $n \rightarrow \infty$ on both sides of the equation above and write

$$
\begin{aligned}
& \lim _{n \rightarrow \infty} \sum_{P_{n}} \beta_{t_{i+1}}\left(\left[\nabla_{\theta} G(X, \theta), \theta\right]_{t_{i+1}}^{x}-\left[\nabla_{\theta} G(X, \theta), \theta\right]_{t_{i}}^{x}\right) \\
& \quad=\left[\int_{\underline{\tau}}^{\bar{\tau}} \beta_{s} \nabla_{\theta} G\left(X_{s}, \theta_{s}\right) d^{*, x} \theta_{s}-\int_{\underline{\tau}}^{\bar{\tau}} \beta_{s} \nabla_{\theta} G\left(X_{s}, \theta_{s}\right) d \theta_{s}\right] \\
& \quad=\left[\beta \nabla_{\theta} G(X, \theta), \theta\right]_{\underline{\tau}}^{\bar{\tau}, x} .
\end{aligned}
$$

The first equality follows from (2.58) and the dominated convergence theorem. The second equality follows from the definition of $\left[\beta \nabla_{\theta} G(X, \theta), \theta\right]_{\underline{\underline{\tau}}}^{\bar{\tau}, x}$. Therefore, (2.52) holds and we rewrite the deviation 
term in (2.3) as follows:

$$
\begin{aligned}
& \Gamma_{k, \gamma}=\int_{\underline{\tau}_{k}}^{\bar{\tau}_{k, \gamma}} \beta_{s} \mathcal{A}_{x, \theta^{*}} G\left(X_{s}, \theta_{s}\right) d s \\
& =\beta_{\bar{\tau}_{k}, \gamma} G\left(X_{\bar{\tau}_{k, \gamma}}, \theta_{\bar{\tau}_{k, \gamma}}\right)-\beta_{\underline{\tau}} G\left(X_{\underline{\tau}}, \theta_{\underline{\tau}}\right)-\int_{\underline{\tau}}^{\bar{\tau}_{k, \gamma}} \beta_{s}^{\prime} G\left(X_{s}, \theta_{s}\right) d s \\
& -\int_{\underline{\tau}}^{\bar{\tau}_{k, \gamma}} \beta_{s} \tilde{G}_{1}\left(X_{s}, \theta_{s}\right) d B_{s}-\int_{\underline{\tau}}^{\bar{\tau}_{k, \gamma}} \int_{\mathbb{R}} \beta_{s} \tilde{G}_{2}\left(X_{s}, \theta_{s}, z\right) \tilde{\mu}(d s, d z) \\
& -\int_{\underline{\tau}}^{\bar{\tau}_{k, \gamma}} \beta_{S} \nabla_{\theta} G\left(X_{S}, \theta_{S}\right) d \theta_{S} \\
& -\left[\int_{\underline{\tau}}^{\bar{\tau}_{k, \gamma}} \frac{1}{2} \beta_{s} \nabla_{\theta}^{2} G_{k}\left(X_{s}, \theta_{s}\right) d[\theta, \theta]_{s}\right]_{k=1}^{d}-\left[\beta \nabla_{\theta} G(X, \theta), \theta\right]_{\underline{\tau}}^{\bar{\tau}_{k, \gamma}, x} .
\end{aligned}
$$

Next, we prove the convergence of each term on the right-hand side of (2.60). The expectation operator under the probability measure $\mathbb{P}_{x_{0}, \theta^{*}}$ is denoted by $\mathbb{E}$. Let $J_{t}^{(1)}=\beta_{t}\left|G\left(X_{t}, \theta_{t}\right)\right|$. The inequality

$$
\begin{aligned}
\mathbb{E}\left[\left|J_{t}^{(1)}\right|^{2}\right] & \leq C_{M, q} \beta_{t}^{2} \mathbb{E}\left[\left(1+\left|\theta_{t}\right|\right)^{2}\left(1+\left|X_{t}\right|^{q}\right)^{2}\right] \\
& \leq 4 C_{M, q} \beta_{t}^{2} \mathbb{E}\left[\left(1+\left|\theta_{t}\right|^{2}\right)\left(1+\left|X_{t}\right|^{2 q}\right)\right] \leq \tilde{C} \beta_{t}^{2},
\end{aligned}
$$

follows from Proposition 2.13, and because the random variables $X_{t}$ and $\theta_{t}$ have finite moments and because $\tilde{C}$ is a constant that depends on $x_{0}$. Now, apply the Borel-Cantelli argument as in Lemma 4.1 in [17] to show that $J_{t}^{(1)}$ converges to zero with probability one. Next, consider the finite variation term on the right-hand side of (2.60)

$$
J_{t, 0}^{(2)}:=\int_{0}^{t}\left|\beta_{s}^{\prime} G\left(X_{s}, \theta_{s}\right)+\beta_{s} \mathcal{L}_{\theta, \theta^{*}} G\left(X_{s}, \theta_{s}\right)\right| d s,
$$

where $\mathcal{L}_{\theta, \theta^{*}}$ is the infinitesimal generator of the process $\theta$ under the probability measure $\mathbb{P}_{\theta^{*}}$. The term $J_{t, 0}^{(2)}$ obeys the bound

$$
\sup _{t>0} \mathbb{E}\left|J_{t, 0}^{(2)}\right| \leq \int_{0}^{\infty} C\left(\left|\beta_{s}^{\prime}\right|+\beta_{s}^{2}\right)\left(1+\mathbb{E}\left|X_{s}\right|^{2 q}+\mathbb{E}\left|\theta_{s}\right|^{2}\right) d s<\infty,
$$

where the last inequality follows from the properties of the learning rate $\beta_{t}$ in Condition 2.4 , and because the process $X$ has a bounded $2 q$-moment and the process $\theta$ has a bounded second moment. Thus, there exists an integrable random variable $J_{\infty, 0}^{(2)}$, such that

$$
J_{t, 0}^{(2)} \rightarrow J_{\infty, 0}^{(2)} \quad \text { as } t \rightarrow \infty \text { with probability one. }
$$

Next, consider the stochastic integral

$$
\begin{aligned}
J_{t, 0}^{(3)}:= & \int_{0}^{t} \beta_{s} \tilde{G}_{1}\left(X_{s}, \theta_{s}\right) d B_{s}+\int_{0}^{t} \int_{\mathbb{R}} \beta_{s} \tilde{G}_{2}\left(X_{s}, \theta_{s}, z\right) \tilde{\mu}(d s, d z) \\
& +\int_{0}^{t} \beta_{s} \nabla_{\theta} G\left(X_{s}, \theta_{s}\right) \nabla_{\theta} b\left(X_{s}, \theta\right) \sigma\left(X_{s}\right)^{-1} d B_{s} .
\end{aligned}
$$


The Burkholder-Davis-Gundy (BDG) inequality implies that

$$
\begin{aligned}
\mathbb{E}\left[\sup _{0 \leq s \leq t}\left|J_{s, 0}^{(3)}\right|^{2}\right] \leq & C_{1} \mathbb{E}\left[\int_{0}^{t}\left|\beta_{s} \tilde{G}_{1}\left(X_{s}, \theta_{s}\right)\right|^{2} d s+\int_{0}^{t} \int_{\mathbb{R}}\left|\beta_{s} \tilde{G}_{2}\left(X_{s}, \theta_{s}, z\right)\right|^{2} v(d z) d s\right. \\
& \left.+\int_{0}^{t}\left|\beta_{s} \nabla_{\theta} G\left(X_{s}, \theta_{s}\right) \nabla_{\theta} b\left(X_{s}, \theta\right) \sigma\left(X_{s}\right)^{-1}\right|^{2} d s\right],
\end{aligned}
$$

where $C_{1}$ is a positive constant. From Lemma 2.14, we have that

$$
\begin{aligned}
\left|\tilde{G}_{1}\left(X_{S}, \theta_{s}\right)\right| & \leq C_{M, q}\left(1+\left|\theta_{s}\right|\right)\left(1+\left|X_{S}\right|^{q}\right) \\
\left|\tilde{G}_{2}\left(X_{S}, \theta_{s}, z\right)\right| & \leq C_{M, q}\left(1+\left|\theta_{s}\right|\right)\left|X_{S}\right||z|\left(1+\left|X_{s}\right|^{q}|z|^{q}+\left|X_{S}\right|^{q}\right) .
\end{aligned}
$$

Therefore, use the BDG and the Cauchy-Schwarz inequalities to show that the expectation of the first and third terms on the right-hand side of (2.62) are both bounded by $C K \int_{0}^{t} \beta_{s}^{2} d s$ because the processes $\theta_{s}$ and $X_{s}$ have finite $p$-moments for all $p>0$. For the remaining term, write

$$
\begin{aligned}
\mathbb{E}\left[\int_{0}^{t} \int_{\mathbb{R}}\left|\beta_{s} \tilde{G}_{2}\left(X_{s}, \theta_{s}, z\right)\right|^{2} v(d z) d s\right] \\
\quad \leq C_{M, q}^{2} \mathbb{E}\left[\int_{0}^{t} \int_{\mathbb{R}} \beta_{s}^{2}\left(1+\left|\theta_{s}\right|^{2}\right)\left|X_{s}\right|^{2}|z|^{2}\left(1+\left|X_{s}\right|^{2 q}|z|^{2 q}+\left|X_{s}\right|^{2 q}\right) v(d z) d s\right] \\
\quad \leq \tilde{C} \mathbb{E}\left[\int_{0}^{t} \beta_{s}^{2}\left(1+\left|\theta_{s}\right|^{2}\right)\left(\left|X_{s}\right|^{2}+\left|X_{s}\right|^{2 q+2}\right) d s\right] \\
\leq 8 \tilde{C} \mathbb{E}\left[\int_{0}^{t} \beta_{s}^{2}\left(1+\left|\theta_{s}\right|^{4}\right)+\beta_{s}^{2}\left(1+\left|X_{s}\right|^{4 q+4}\right) d s\right]<\hat{C} \int_{0}^{t} \beta_{s}^{2} d s,
\end{aligned}
$$

where $\tilde{C}$ and $\hat{C}$ are positive constants that depend on $x_{0}$. The first inequality in (2.64) results from Lemma 2.14, the second inequality follows from Condition 2.3, and the last inequality follows because the processes $X_{t}$ and $\theta_{t}$ have finite moments, see Lemma 2.8. Hence, the right-hand side of (2.62) is finite. Then, by Doob's martingale convergence theorem, there exists a square integrable random variable $J_{\infty, 0}^{(3)}$, such that

$$
J_{t, 0}^{(3)} \rightarrow J_{\infty, 0}^{(3)} \quad \text { as } t \rightarrow \infty \text { with probability one. }
$$

Next, consider the following covariation in the interval $\left[t_{i}, t_{i+1}\right]$

$$
\begin{aligned}
& \left|\left[\beta \nabla_{\theta} G(X, \theta), \theta\right]_{t_{i}}^{t_{i+1}, x}\right| \\
& \leq \lim _{\left\|\tilde{P}_{n}\right\| \rightarrow 0}\left(\sum_{\tilde{P}_{n}} \beta_{s_{j+1}}\left|\nabla_{\theta} G\left(X_{s_{j+1}}, \theta_{s_{j}}\right)-\nabla_{\theta} G\left(X_{s_{j}}, \theta_{s_{j}}\right)\right|\left|\theta_{s_{j+1}}-\theta_{s_{j}}\right|\right. \\
& \left.\quad+\left|\beta_{s_{j}}-\beta_{s_{j+1}}\right|\left|\nabla_{\theta} G\left(X_{s_{j}}, \theta_{s_{j}}\right)\right|\left|\theta_{s_{j+1}}-\theta_{s_{j}}\right|\right)
\end{aligned}
$$


where $\tilde{P}_{n}$ is the partition of the interval $\left[t_{i}, t_{i+1}\right]$. Now, consider the second term on the right-hand side of (2.66)

$$
\begin{aligned}
& \lim _{\left\|\tilde{P}_{n}\right\| \rightarrow 0} \sum_{\tilde{P}_{n}}\left|\beta_{s_{j}}-\beta_{s_{j+1}}\right|\left|\nabla_{\theta} G\left(X_{s_{j}}, \theta_{s_{j}}\right)\right|\left|\theta_{s_{j+1}}-\theta_{s_{j}}\right| \\
& \quad \leq \lim _{\left\|\tilde{P}_{n}\right\| \rightarrow 0} \sum_{\tilde{P}_{n}} C_{G, q}\left|\beta_{s_{j}}-\beta_{s_{j+1}}\right|\left(1+\sup _{t_{i}<s<t_{i+1}}\left|X_{s}\right|^{q}\right) \sup _{j}\left|\theta_{s_{j+1}}-\theta_{s_{j}}\right| \\
& \quad \leq \lim _{\left\|\tilde{P}_{n}\right\| \rightarrow 0} C_{G, q}\left(1+\sup _{t_{i}<s<t_{i+1}}\left|X_{s}\right|^{q}\right)\left|\beta_{t_{i}}-\beta_{t_{i+1}}\right| \sup _{j}\left|\theta_{s_{j+1}}-\theta_{s_{j}}\right|=0,
\end{aligned}
$$

where the first inequality follows from (2.18) and the last inequality follows because the process $\theta_{s}$ is continuous. Now, consider the term

$$
\begin{aligned}
& \lim _{\left\|\tilde{P}_{n}\right\| \rightarrow 0} \sum_{\tilde{P}_{n}} \beta_{s_{j+1}}\left|\nabla_{\theta} G\left(X_{s_{j+1}}, \theta_{s_{j}}\right)-\nabla_{\theta} G\left(X_{s_{j}}, \theta_{s_{j}}\right)\right|\left|\theta_{s_{j+1}}-\theta_{s_{j}}\right| \\
& \quad \leq \lim _{\left\|\tilde{P}_{n}\right\| \rightarrow 0} \sum_{t_{i}<s_{j}<t_{i+1}} C_{G, q} \beta_{s_{j+1}}\left(1+\sup _{t_{i}<s<t_{i+1}}\left|X_{s}\right|^{q}\right)\left|X_{s_{j+1}}-X_{s_{j}}\right|\left|\theta_{s_{j+1}}-\theta_{s_{j}}\right| \\
& \quad \leq C_{G, q} \beta_{t_{i}}\left(1+\sup _{t_{i}<s<t_{i+1}}\left|X_{s}\right|^{q}\right) \int_{t_{i}}^{t_{i+1}}\left|d[X, \theta]_{s}\right| \\
& \quad=C_{G, q} \beta_{t_{i}}^{2}\left(1+\sup _{t_{i}<s<t_{i+1}}\left|X_{S}\right|^{q}\right) \int_{t_{i}}^{t_{i+1}}\left|\nabla_{\theta} b\left(X_{s}, \theta_{s}\right)\right| d s,
\end{aligned}
$$

where the first inequality follows from (2.18) and the second inequality follows from the definition of covariation. Therefore, for $t>r$, from (2.68), we take the sum and the expectation on the left-hand side of (2.66) to obtain

$$
\begin{aligned}
& \mathbb{E}\left[\left|\left[\beta \nabla_{\theta} G(X, \theta), \theta\right]_{r}^{t, x}\right|\right] \\
& \leq C_{G, q} \sum_{i=\lfloor r\rfloor}^{\infty} \beta_{i}^{2} \mathbb{E}\left[\left(1+\sup _{i<s<i+1}\left|X_{s}\right|^{q}\right) \int_{i}^{i+1}\left|\nabla_{\theta} b\left(X_{s}, \theta_{s}\right)\right| d s\right] \\
& \quad \leq C_{G, q} \sum_{i=\lfloor r\rfloor}^{\infty} \beta_{i}^{2} \mathbb{E}\left[\left(1+\sup _{i<s<i+1}\left|X_{s}\right|^{2 q}\right)\right] \mathbb{E}\left[\int_{i}^{i+1}\left|\nabla_{\theta} b\left(X_{s}, \theta_{s}\right)\right|^{2} d s\right] \\
& \leq C_{G, q} \sum_{i=\lfloor r\rfloor}^{\infty} \beta_{i}^{2}(1+\sqrt{(1+i)})\left[\int_{i}^{i+1} \mathbb{E}\left|\nabla_{\theta} b\left(X_{s}, \theta_{s}\right)\right|^{2} d s\right] \\
& \quad \leq C_{G, q} \sum_{i=\lfloor r\rfloor}^{\infty} \beta_{i}^{2}(1+\sqrt{(1+i)})<\frac{\tilde{C}}{\sqrt{1+r}}
\end{aligned}
$$

where the second inequality follows from the Cauchy -Schwarz inequality, the third inequality follows from Lemma 4.1 in the online supplement [2], and the last inequality follows from Condition 2.5 on $\nabla_{\theta} b(x, \theta)$ and because $\mathbb{E}\left|X_{S}\right|^{2 q}$ is finite. The inequality above implies that there exists a finite random 
variable $J_{\infty, 0}$, such that

$$
\left|\left[\beta \nabla_{\theta} G(X, \theta), \theta\right]_{0}^{t, x}\right| \rightarrow J_{\infty, 0} \quad \text { as } t \rightarrow \infty
$$

with probability one. From (2.60), we write that

$$
\left|\Gamma_{k, \gamma}\right| \leq J_{\bar{\tau}_{k, \gamma}}^{(1)}+J_{\underline{\tau}_{k}}^{(1)}+J_{\bar{\tau}_{k, \gamma}, \underline{\tau}_{k}}^{(2)}+\left|J_{\bar{\tau}_{k, \gamma}, \underline{\tau}_{k}}^{(3)}\right|+\left|\left[\beta \nabla_{\theta} G(X, \theta), \theta\right]_{\underline{\tau}_{k}}^{\bar{\tau}_{k, \gamma}, x}\right| .
$$

From (2.61), (2.65), (2.69), each term on the right-hand side converges to 0 as $k \rightarrow \infty$, which implies that $\left|\Gamma_{k, \gamma}\right| \rightarrow 0$ as $k \rightarrow \infty$.

\subsection{Convergence of jump SGDCT}

In this subsection, we prove a sequence of lemmas and show the convergence of the estimator in (1.9) to a stationary point of the function $\bar{g}$; we do so in two steps. First, we analyse the deviation term $\Gamma_{k, \gamma}$ in (2.3) when $k \rightarrow \infty$. In our case, the jump-diffusion process $X$ is Markov, therefore we need to use the ergodicity of the process $X$ to quantify the deviation term $\Gamma_{k, \gamma}$ via the Poisson equation in (2.12), see Pardoux and Veretennikov [15]. The technique dates back to Delyon [5] and Metivier and Priouret [13].

In Proposition 2.16, we showed that the absolute value of the deviation term $\Gamma_{k, \gamma}$ is asymptotically close to zero. Below, we use a similar argument as that in Proposition 3 in [1] to prove that $\lim _{t \rightarrow \infty}\left|\nabla_{\theta} \bar{g}\left(\theta_{t}\right)\right|=0$ almost surely. For a proof of the following two lemmas, see Section 4 in the online supplement [2].

Lemma 2.17. Suppose Conditions 2.1 to 2.6 hold. Choose a constant $\tilde{\lambda}$, such that for a given $\kappa>0$ we have $3 \tilde{\lambda}+\tilde{\lambda} / 4 \kappa=1 / 2 L_{\nabla \bar{g}}$, where $L_{\nabla \bar{g}}$ is the Lipschitz constant of $\nabla \bar{g}$. For $k$ large enough and for $\eta>0$ small enough, we have

$$
\int_{\underline{\tau}_{k}}^{\bar{\tau}_{k, \eta}} \beta_{s} d s>\tilde{\lambda} \quad \text { and } \tilde{\lambda} / 2 \leq \int_{\underline{\tau}_{k}}^{\bar{\tau}_{k}} \beta_{s} d s \leq \tilde{\lambda} .
$$

The following lemma shows that the value of the function $\bar{g}$ decreases when the value of $k$ is large enough.

Lemma 2.18. Suppose Conditions 2.1 to 2.6 hold and assume that there are an infinite number of intervals $I_{k}=\left[\underline{\tau}_{k}, \bar{\tau}_{k}\right)$. Then, there is a fixed constant $\gamma>0$, such that for $k$ large enough, we have

$$
\bar{g}\left(\theta_{\bar{\tau}_{k}}\right)-\bar{g}\left(\theta_{\underline{\tau}_{k}}\right) \leq-\gamma .
$$

Next, we show that the value of $\bar{g}\left(\theta_{\underline{\tau}_{k}}\right)-\bar{g}\left(\theta_{\bar{\tau}_{k-1}}\right)$ eventually starts to decrease as $k \rightarrow \infty$.

Lemma 2.19. Suppose Conditions 2.1 to 2.6 hold and assume that there is an infinite number of intervals $I_{k}=\left[\underline{\tau}_{k}, \bar{\tau}_{k}\right)$. There is a fixed constant $\gamma_{1}<\gamma$, such that for $k$ large enough, one has

$$
\bar{g}\left(\theta_{\underline{\tau}_{k}}\right)-\bar{g}\left(\theta_{\bar{\tau}_{k-1}}\right) \leq \gamma_{1} .
$$

Proof. See Lemma 3.5 in [17].

The next theorem shows the convergence of the SGDCT estimator. 
Theorem 2.20 (Convergence of the gradient). Let the process $\theta_{t}$ follow (1.9) and assume Conditions 2.1 to 2.6 hold. Then

$$
\left|\nabla_{\theta} \bar{g}\left(\theta_{t}\right)\right| \rightarrow 0 \quad \text { as } t \rightarrow \infty \text {, almost surely. }
$$

Proof. Apply Lemmas 2.18 and 2.19 above, and Theorem 2.4 in [17].

The crucial part of the proof of the convergence of the SGDCT estimator is the convergence of the deviation term $\Gamma_{k, \gamma}$ in (2.3), which we proved in Proposition 2.16.

\section{Conclusions and future research}

In this paper, we showed the convergence of the SGDCT algorithm to estimate the drift parameter of a jump-diffusion process. Our paper is an extension to the work of Sirignano and Spiliopoulos [17]. We proposed two estimators: an estimator that uses the raw data, and another that filters the jumps of the jump-diffusion process before estimating the drift parameter.

Our estimators are desirable because online updates are more computationally efficient than offline updates. As new observations arrive, the online estimator is updated, while offline algorithms solve a new optimisation problem to estimate the unknown parameter.

We employed simulations to illustrate the performance of the estimators. We see that when the agent filters the jumps in the process, the estimator converges quicker than when the agent employs the raw data (i.e., without filtering jumps).

The SGDCT estimator is well-suited for the recent advances in control theory that focus on adaptive robust control to obtain strategies robust to model uncertainty, see Bielecki et al. [3]. The adaptive control framework requires an estimator that is continuously updated with the arrival of new information and also requires that the coupled process $\left(X_{t}, \theta_{t}\right)_{t \geq 0}$ is Markov. The SGDCT algorithm we developed here meets both requirements.

The online estimators may be employed in many financial problems where prices, or other observed variables, follow jump-diffusion processes. For example, in high-frequency trading, many models assume that the midprice follows, over a short-period of time, an OU-type process. A similar application is in pairs trading, where a linear combination of two or more assets follows an OU-type process, see Cartea et al. [4]. In both cases, the success of these high-frequency trading strategies relies on being able to estimate the drift of a process. An important advantage of our estimator is that the estimates of the drift are quickly updated with the arrival of every innovation in the prices of the assets, so the strategies can be implemented in real time.

Finally, there are a number of future research directions. For example, one may analyse the statistical properties of the jump-diffusion SGDCT we presented here. If the function $b$ is convex, one could follow the same argument as that Sirignano and Spiliopoulos [18] to prove the central limit theorem of the estimator (1.9). Although most of the proof of this extension would follow from standard results on the property of jump-diffusion processes, one would still require the proof of the convergence of the term (2.2). Also, it would be interesting to study the jump-diffusion filtering problem where the agent partially observes the process.

\section{Supplementary Material}

Supplement to “Online drift estimation for jump-diffusion processes” (DOI: 10.3150/20-BEJ1319 SUPP; .pdf). The supplementary material in [2] contains three sections. The first section proposes 
an alternative SGDCT estimator that employs the continuous part of the process $X$ to estimate the unknown parameter $\theta^{*}$. The second section discusses the performance of the SGDCT estimator. The last section provides proofs of Lemmas 2.8, 2.11, 2.12, 2.17, 2.18, and additional results and theorems, and mathematical notation.

\section{References}

[1] Bertsekas, D.P. and Tsitsiklis, J.N. (2000). Gradient convergence in gradient methods with errors. SIAM J. Optim. 10 627-642. MR1741189 https://doi.org/10.1137/S1052623497331063

[2] Bhudisaksang, T. and Cartea, Á. (2021). Supplement to "Online drift estimation for jump-diffusion processes." https://doi.org/10.3150/20-BEJ1319SUPP

[3] Bielecki, T., Chen, T. and Cialenco, I. (2017). Recursive construction of confidence regions. Electron. J. Stat. 11 4674-4700. MR3724972 https://doi.org/10.1214/17-EJS1362

[4] Cartea, Á., Jaimungal, S. and Penalva, J. (2015). Algorithmic and High-Frequency Trading. Cambridge: Cambridge Univ. Press. MR0838085

[5] Delyon, B. (1996). General results on the convergence of stochastic algorithms. IEEE Trans. Automat. Control 41 1245-1255. MR1409470 https://doi.org/10.1109/9.536495

[6] Errami, M., Russo, F. and Vallois, P. (2002). Itô's formula for $C^{1, \lambda}$-functions of a càdlàg process and related calculus. Probab. Theory Related Fields 19 535-552. MR1894067

[7] Föllmer, H., Protter, P. and Shiryayev, A.N. (1995). Quadratic covariation and an extension of Itô's formula. Bernoulli 1 149-169. MR1354459 https://doi.org/10.2307/3318684

[8] Kessler, M. (1997). Estimation of an ergodic diffusion from discrete observations. Scand. J. Stat. 24 211-229. MR1455868 https://doi.org/10.1111/1467-9469.00059

[9] Kunita, H. (2004). Representation of martingales with jumps and applications to mathematical finance. In Stochastic Analysis and Related Topics in Kyoto. Adv. Stud. Pure Math. 41 209-232. Tokyo: Math. Soc. Japan. MR2083711 https://doi.org/10.2969/aspm/04110209

[10] Kutoyants, Y.A. (2013). Statistical Inference for Ergodic Diffusion Processes. Berlin: Springer.

[11] Levanony, D., Shwartz, A. and Zeitouni, O. (1994). Recursive identification in continuous-time stochastic processes. Stochastic Process. Appl. 49 245-275. MR1260193 https://doi.org/10.1016/0304-4149(94) 90137-6

[12] Masuda, H. (2007). Ergodicity and exponential $\beta$-mixing bounds for multidimensional diffusions with jumps. Stochastic Process. Appl. 117 35-56. MR2287102 https://doi.org/10.1016/j.spa.2006.04.010

[13] Métivier, M. and Priouret, P. (1984). Applications of a Kushner and Clark lemma to general classes of stochastic algorithms. IEEE Trans. Inf. Theory 30 140-151. MR0807052 https://doi.org/10.1109/TIT.1984. 1056894

[14] Pardoux, E. and Veretennikov, A.Y. (2001). On the Poisson equation and diffusion approximation. I. Ann. Probab. 29 1061-1085. MR1872736 https://doi.org/10.1214/aop/1015345596

[15] Pardoux, È. and Veretennikov, A.Y. (2003). On Poisson equation and diffusion approximation. II. Ann. Probab. 31 1166-1192. MR1988467 https://doi.org/10.1214/aop/1055425774

[16] Protter, P. (1990). Stochastic Integration and Differential Equations: A New Approach. Applications of Mathematics (New York) 21. Berlin: Springer. MR1037262 https://doi.org/10.1007/978-3-662-02619-9

[17] Sirignano, J. and Spiliopoulos, K. (2017). Stochastic gradient descent in continuous time. SIAM J. Financial Math. 8 933-961. MR3732944 https://doi.org/10.1137/17M1126825

[18] Sirignano, J. and Spiliopoulos, K. (2020). Stochastic gradient descent in continuous time: A central limit theorem. Stoch. Syst. 10 124-151. MR4119247 https://doi.org/10.1287/stsy.2019.0050

[19] Sørensen, M. (1991). Likelihood methods for diffusions with jumps. In Statistical Inference in Stochastic Processes. Probab. Pure Appl. 6 67-105. New York: Dekker. MR1138259

[20] Surace, S.C. and Pfister, J.-P. (2019). Online maximum-likelihood estimation of the parameters of partially observed diffusion processes. IEEE Trans. Automat. Control 64 2814-2829. MR3978276

[21] Uehara, Y. (2019). Statistical inference for misspecified ergodic Lévy driven stochastic differential equation models. Stochastic Process. Appl. 129 4051-4081. MR3997671 https://doi.org/10.1016/j.spa.2018.11.007 
[22] Veretennikov, A. and Kulik, A. (2012). Extended Poisson equation for weakly ergodic Markov processes. Theory Probab. Math. Statist. 85 23-39. MR2933700

[23] Wang, J. (2010). Regularity of semigroups generated by Lévy type operators via coupling. Stochastic Process. Appl. 120 1680-1700. MR2673970 https://doi.org/10.1016/j.spa.2010.04.007

[24] Yoshida, N. (1992). Estimation for diffusion processes from discrete observation. J. Multivariate Anal. 41 220-242. MR1172898 https://doi.org/10.1016/0047-259X(92)90068-Q

Received April 2020 and revised November 2020 